\title{
Excess Copper-Induced Alterations of Protein Profiles and Related Physiological Parameters in Citrus Leaves
}

\author{
Wei-Lin Huang ${ }^{1}$, Feng-Lin Wu ${ }^{1}$, Hui-Yu Huang ${ }^{1}$, Wei-Tao Huang ${ }^{1}$, Chong-Ling Deng ${ }^{2}$, \\ Lin-Tong Yang ${ }^{1} \mathbb{D}$, Zeng-Rong Huang ${ }^{2}$ and Li-Song Chen $1,3,4, * \mathbb{D}$ \\ 1 Institute of Plant Nutritional Physiology and Molecular Biology, College of Resources and Environment, \\ Fujian Agriculture and Forestry University, Fuzhou 350002, China; 1170807014@fafu.edu.cn (W.-L.H.); \\ 1180807019@fafu.edu.cn (F.-L.W.); 1180807011@fafu.edu.cn (H.-Y.H.); 1190807012@fafu.edu.cn (W.-T.H.); \\ talstoy@fafu.edu.cn (L.-T.Y.) \\ 2 Guangxi Key Laboratory of Citrus Biology, Guangxi Academy of Specialty Crops, Guilin 541004, China; \\ cldeng88168@126.com (C.-L.D.); huangzengrong@fafu.edu.cn (Z.-R.H.) \\ 3 Fujian Provincial Key Laboratory of Soil Environmental Health and Regulation, College of Resources and \\ Environment, Fujian Agriculture and Forestry University, Fuzhou 350002, China \\ 4 The Higher Education Key Laboratory of Fujian Province for Soil Ecosystem Health and Regulation, \\ College of Resources and Environment, Fujian Agriculture and Forestry University, Fuzhou 350002, China \\ * Correspondence: lisongchen@fafu.edu.cn or lisongchen2002@hotmail.com; Tel.: +86-591-8385-4966
}

Received: 12 February 2020; Accepted: 24 February 2020; Published: 28 February 2020

check for updates

\begin{abstract}
This present study examined excess copper $(\mathrm{Cu})$ effects on seedling growth, leaf $\mathrm{Cu}$ concentration, gas exchange, and protein profiles identified by a two-dimensional electrophoresis (2-DE) based mass spectrometry (MS) approach after Citrus sinensis and Citrus grandis seedlings were treated for six months with 0.5 (control), 200, 300, or $400 \mu \mathrm{M} \mathrm{CuCl}_{2}$. Forty-one and 37 differentially abundant protein (DAP) spots were identified in Cu-treated C. grandis and C. sinensis leaves, respectively, including some novel DAPs that were not reported in leaves and/or roots. Most of these DAPs were identified only in C. grandis or C. sinensis leaves. More DAPs increased in abundances than DAPs decreased in abundances were observed in Cu-treated C. grandis leaves, but the opposite was true in Cu-treated C. sinensis leaves. Over $50 \%$ of DAPs were associated with photosynthesis, carbohydrate, and energy metabolism. Cu-toxicity-induced reduction in leaf $\mathrm{CO}_{2}$ assimilation might be caused by decreased abundances of proteins related to photosynthetic electron transport chain (PETC) and $\mathrm{CO}_{2}$ assimilation. $\mathrm{Cu}$-effects on PETC were more pronounced in $C$. sinensis leaves than in C. grandis leaves. DAPs related to antioxidation and detoxification, protein folding and assembly (viz., chaperones and folding catalysts), and signal transduction might be involved in Citrus Cu-toxicity and Cu-tolerance.
\end{abstract}

Keywords: Citrus grandis; Citrus sinensis; $\mathrm{CO}_{2}$ assimilation; copper-toxicity; 2-DE; leaves

\section{Introduction}

Microelement copper $(\mathrm{Cu})$ is highly toxic to plants when in excess. $\mathrm{Cu}$-containing fungicides and bactericides are widely used in agriculture to control fungal and bacterial diseases in crops including Citrus in order to improve crop production and quality. Cu contamination in agriculture soils is on the rise all over the world [1,2]. Cu accumulation in soils can cause Cu-toxicity and related nutritional disorders, resulting in a series of adverse effects on plants ranging from morphological and physiological to molecular levels [1,3]. In old Citrus orchards, the excess accumulation of $\mathrm{Cu}$ in soils is a common phenomenon because of the extensive and continued use of $\mathrm{Cu}$-containing agricultural chemicals against fruit and foliar diseases such as anthracnose and canker [3,4]. Cu concentration and availability in soils under continuous Citrus production orchards increase with increasing production 
period [2]. In Citrus, the common Cu-toxic symptoms include leaf iron (Fe) chlorosis, poor growth, and stunted, and discolored root systems [3,5].

$\mathrm{Cu}$, which can act as a cofactor for over 100 proteins including plastocyanin, laccase, cytochrome $c$ oxidase, $\mathrm{Cu} / \mathrm{zinc}(\mathrm{Zn})$ superoxide dismutase (SOD), ethylene receptors, amino oxidase, polyphenol oxidases, ascorbate (ASC) oxidase, diamine oxidases, and phytocyanin, is involved in photosynthesis, respiration, ATP biosynthesis, ethylene reception, reactive oxygen species (ROS) metabolism, cell wall formation, and carbon, lipid, and nitrogen metabolisms [6]. Accordingly, a lot of researchers have examined the toxic effects of $\mathrm{Cu}$ on the uptake of nutrients and water [1,5], growth [1,3], photosynthetic pigment production [7], photosynthetic electron transport [5,8], $\mathrm{CO}_{2}$ assimilation [8], carbohydrate and nitrogen $(\mathrm{N})$ metabolism [7,9], respiration [10], hormonal status [11], cell wall metabolism [12], phenolic metabolism [13], as well as ROS generation and detoxification [8].

Although $\mathrm{Cu}$-toxic effects on plant growth and physiology have been investigated in some details [2,14], little is known about Cu-toxicity-induced alteration of protein profiles in plants. Proteomics is a powerful approach to elucidate the complicated responses of plants to unfavorable environments $[15,16]$. Recently, there have been several reports investigating $\mathrm{Cu}$ toxicity responsive proteins. Most reports, however, have focused on herbaceous plants, including rice [17-19], Allium cepa [20], Oenothera glazioviana [21], Arabidopsis [22], Cannabis sativa [23], Agrostis capillaris [24], Elsholtzia splendens $[25,26]$, sorghum $[27,28]$ and wheat [29], while only one study investigated Cu-toxic effects on protein profiles in leaves of woody plant Eucalyptus camaldulensis [30]. Also, most of the above studies mainly focused on Cu-toxicity-responsive proteins occurring in roots because $\mathrm{Cu}$ is preferentially accumulated in Cu-stressed roots, while only few studies investigated Cu-toxic effects on protein profiles in leaves $[25,27,29,30]$. Evidence shows that the toxic effects of $\mathrm{Cu}$ on plant proteomics vary with $\mathrm{Cu}$ concentration, plant species, populations and/or cultivars, and plant tissues $[17,18,24,25,27-29]$. Therefore, more extensive proteomic research on the leaves of woody plants is needed to elucidate the molecular mechanisms of plants under $\mathrm{Cu}$-toxicity.

Here, a two-dimensional electrophoresis (2-DE) based mass spectrometry (MS) approach was used to examine $\mathrm{Cu}$-toxicity-responsive proteins in Citrus grandis and Citrus sinensis leaves. Meanwhile, we examined excess $\mathrm{Cu}$ effects on seedling growth, and leaf $\mathrm{Cu}$ concentration and gas exchange. The objectives were (a) to identify $\mathrm{Cu}$ toxicity responsive proteins in Citrus leaves and (b) to screen the candidate proteins possibly responsible for $\mathrm{Cu}$ tolerance in Citrus.

\section{Results}

\subsection{Excess Cu-Effects on Seedling Growth, Leaf $\mathrm{Cu}$ and Gas Exchange}

As shown in Supplementary Figures S1 and S2, C. sinensis (C. grandis) biomass remained little changed as $\mathrm{Cu}$ concentration in the nutrient solution elevated from 0.5 to $300(200) \mu \mathrm{M}$, then declined at 400 (300-400) $\mu \mathrm{M} \mathrm{Cu}$. Biomass was lower in C. sinensis seedlings than that in C. grandis seedlings at each given $\mathrm{Cu}$ supply.

Leaf $\mathrm{Cu}$ concentration increased with $\mathrm{Cu}$ supply and did not differ between the two Citrus species with the exception that its concentration in leaves was higher in C. sinensis than that in C. grandis at $300 \mu \mathrm{M}$ (Figure 1A).

Leaf $\mathrm{CO}_{2}$ concentration and stomatal conductance $\left(\mathrm{g}_{\mathrm{s}}\right)$ kept unchanged or increased as $\mathrm{Cu}$ concentration in the nutrient solution rose from 0.5 to $200 \mu \mathrm{M}$, then declined with further rise in $\mathrm{Cu}$ concentration. $\mathrm{Cu}$ supply had little influence on the ratio of intercellular to ambient $\mathrm{CO}_{2}$ concentration $\left(C_{i} / C_{a}\right)$ except for that $C_{i} / C_{a}$ in $C$. grandis leaves was slightly higher at $200 \mu \mathrm{M} \mathrm{Cu}$ than that at $300-400$ $\mu \mathrm{M} \mathrm{Cu}$. No significant differences were observed in the three parameters between the two Citrus species over the range of Cu supply (Figure 1B-D).

Based on these results, seedlings that received $300-400 \mu \mathrm{M} \mathrm{Cu}$ were regarded as $\mathrm{Cu}$ excess. 


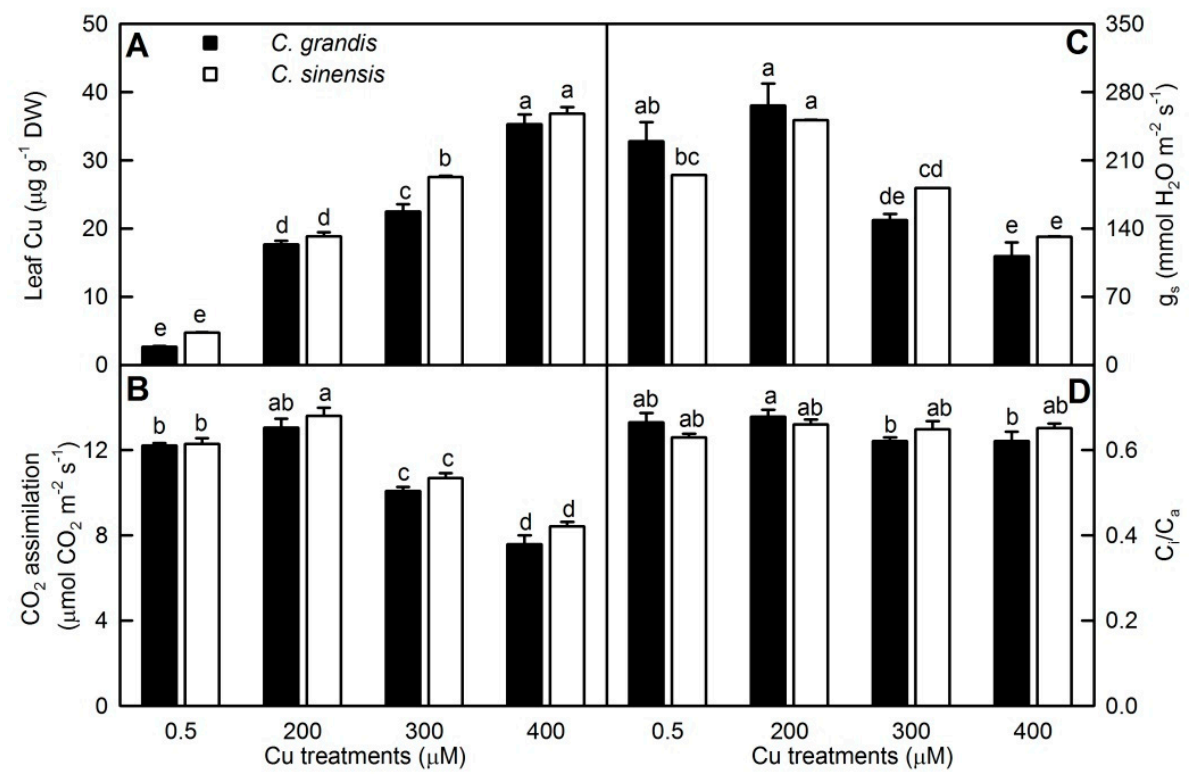

Figure 1. Cu-effects on $\mathrm{Cu}$ concentration (A), $\mathrm{CO}_{2}$ assimilation $(\mathbf{B})$, stomatal conductance $\left(\mathrm{g}_{\mathrm{s}}, \mathrm{C}\right)$ and ratio of intercellular to ambient $\mathrm{CO}_{2}$ concentration $\left(\mathrm{C}_{\mathrm{i}} / \mathrm{C}_{\mathrm{a}}, \mathrm{D}\right)$ in Citrus grandis and Citrus sinensis leaves. Bars represent means \pm SE ( $n=8$ except for 4 for leaf $\mathrm{Cu}$ ). Different letters above the bars indicate significant differences at $p<0.05$.

\subsection{Protein Yield and Cu-responsive Proteins in Leaves}

Three biological replicates were performed in order to obtain reliable data. No significant differences were observed in protein yields and the number of protein spots per gel among eight means (Table 1, Figure 2, Supplementary Figures S3 and S4).

As shown in Tables 1 and 2, Supplementary Table S1 and Figure S5, a total of 42 and 45 differentially abundant protein (DAP) spots were obtained from Cu-treated C. grandis and C. sinensis leaves, respectively. All of these DAP spots were submitted to matrix-assisted laser desorption/ionization tandem time-of-flight mass spectrometry (MALDI-TOF/TOF-MS) based identification. In total, 41 and 37 DAP spots were identified in 200, 300, and/or $400 \mu \mathrm{M}$ Cu-treated C. sinensis and C. grandis leaves, responsively. Most of these DAP spots only presented in Cu-treated C. sinensis or C. grandis leaves. Only seven DAPs with the same accession number [viz., Orange1.1t05091.1, S-adenosyl-L-homocysteine (AdoHcy) hydrolase (Orange1.1t01892.1), chaperonin CPN60-1, (Orange1.1t01459.2), major allergen Pru ar 1 (Cs9g03630.1), ribulose bisphosphate carboxylase/oxygenase (Rubisco) activase 1 (Cs7g31800.3), sedoheptulose-1,7-bisphosphatase (Cs7g31640.4), and 29 kDa ribonucleoprotein A (CP29A; Cs6g11900.1)] presented in the two Citrus species. Fifteen, 12 and 12 (2, 4, and 5) spots increased in abundances and 4, 2, and $9(9,12$, and 23) spots decreased (including disappeared) in abundances were identified in 200, 300, or $400 \mu \mathrm{M} \mathrm{Cu}$-treated C. grandis (C. sinensis) leaves, responsively. Obviously, more (less) DAPs increased in abundances than DAPs decreased in abundances were obtained in 200, 300, or $400 \mu \mathrm{M}$ Cu-treated C. grandis (C. sinensis) leaves. For C. grandis, 10, 6, or 14 DAP spots were identified only in 200, 300 or $400 \mu \mathrm{M}$ Cu-treated leaves, respectively, only 2 DAP spots with the same accession number (viz., malate dehydrogenase (MDH, Cs9g10470.1) and glutathione S-transferase (GST, Cs5g32800.1)) were shared by the three. For C. sinensis, 3, 3, or 15 DAP spots were identified only in 200, 300, or $400 \mu \mathrm{M}$ Cu-treated leaves, respectively, only 2 DAP spots with the same accession number (viz., ferritin-3 (Cs6g09150.2) and enolase (Cs6g15540.1)) were shared by the three. 


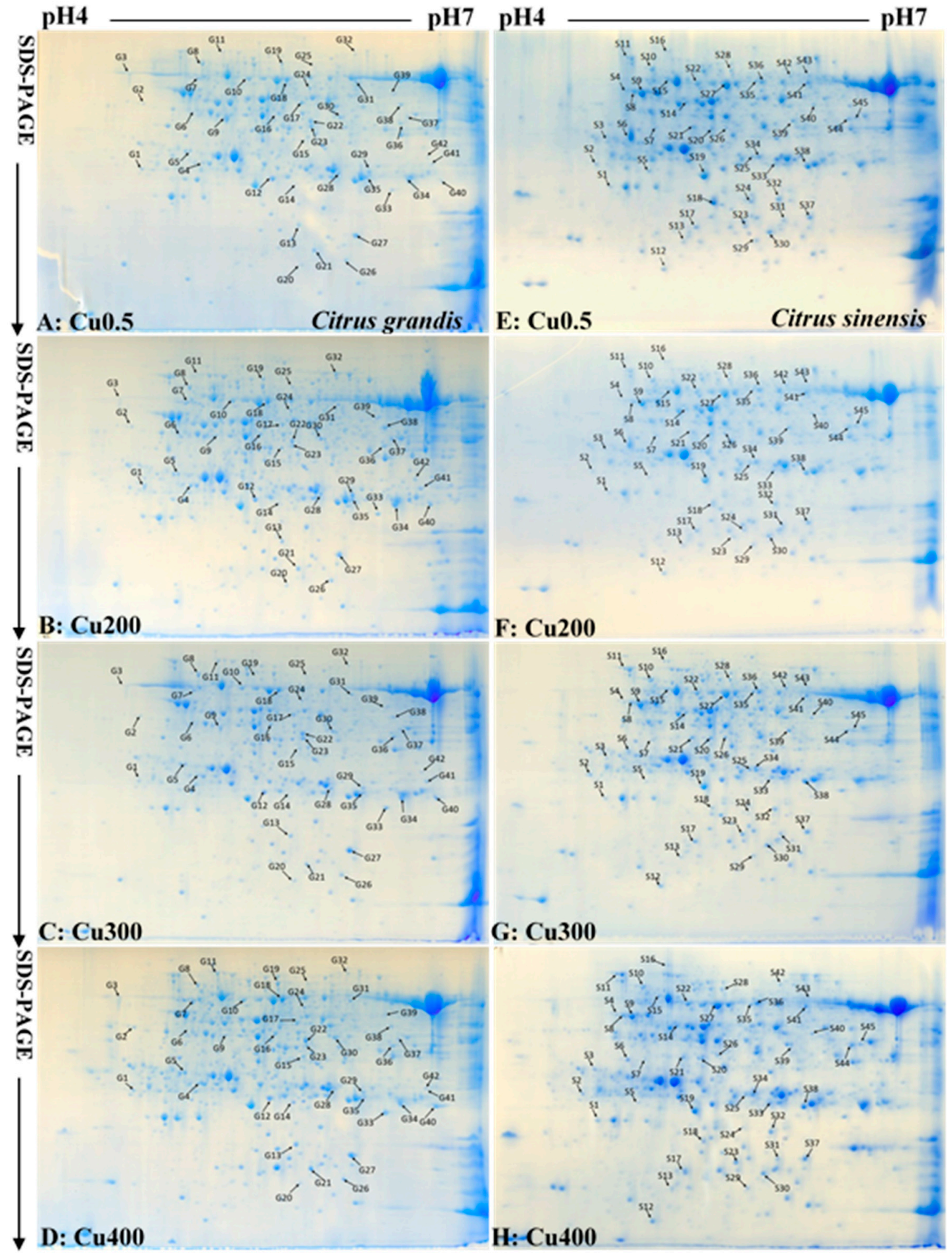

Figure 2. Representative 2-DE images of proteins extracted from $0.5(\mathbf{A}, \mathbf{E}), 200(\mathbf{B}, \mathbf{F}) 300(\mathbf{C}, \mathbf{G})$ and 400 $(\mathbf{D}, \mathbf{H}) \mathrm{Cu}-$ treated Citrus grandis (A-D) and Citrus sinensis $(\mathbf{E}-\mathbf{H})$ leaves. 
Table 1. Protein yield, number of differentially abundant protein (DAP) spots and number of identified DAP spots in 0.5 (control), 200 (Cu200), 300 (Cu300) or 400 (Cu400) Cu-treated Citrus grandis and C. sinensis leaves.

\begin{tabular}{|c|c|c|c|c|c|c|c|c|c|c|}
\hline & \multicolumn{5}{|c|}{ Citrus grandis } & \multicolumn{5}{|c|}{ Citrus sinensis } \\
\hline & Control & Cu200 & Cu300 & $\mathrm{Cu} 400$ & Total & Control & Cu200 & Cu300 & $\mathrm{Cu} 400$ & Total \\
\hline Protein yield $\left(\mathrm{mg} \mathrm{g}^{-1} \mathrm{DW}\right)$ & $49.4 \pm 5.1 \mathrm{a}$ & $45.3 \pm 0.7 \mathrm{a}$ & $47.6 \pm 1.2 \mathrm{a}$ & $44.7 \pm 8.3 a$ & & $44.4 \pm 5.0 \mathrm{a}$ & $43.8 \pm 5.4 a$ & $40.9 \pm 5.4 a$ & $35.6 \pm 3.1 \mathrm{a}$ & \\
\hline $\begin{array}{l}\text { Number of spots per gel } \\
\text { Number of DAP spots }\end{array}$ & $613 \pm 4 a$ & $627 \pm 8 \mathrm{a}$ & $621 \pm 12 \mathrm{a}$ & $621 \pm 22 \mathrm{a}$ & & $614 \pm 7 \mathrm{a}$ & $625 \pm 15 a$ & $617 \pm 12 \mathrm{a}$ & $618 \pm 9 a$ & \\
\hline Increased in abundances & & 15 & 12 & 13 & & & 2 & 4 & 5 & \\
\hline Decreased in abundances & & 4 & 2 & 7 & & & 8 & 12 & 18 & \\
\hline Disappeared & & & & 2 & & & 2 & 1 & 13 & \\
\hline Total & & 19 & 14 & 22 & 42 & & 12 & 17 & 36 & 45 \\
\hline \multicolumn{11}{|l|}{ Number of identified DAP spots } \\
\hline Increased in abundances & & 15 & 12 & 12 & & & 2 & 4 & 5 & \\
\hline Decreased in abundances & & 4 & 2 & 7 & & & 7 & 11 & 12 & \\
\hline Disappeared & & & & 2 & & & 2 & 1 & 11 & \\
\hline Total & & 19 & 14 & 21 & 41 & & 11 & 16 & 28 & 37 \\
\hline
\end{tabular}

Note: Means $( \pm \mathrm{SE}, n=3)$ with a row followed by the same letter are not significant different at $p<0.05$. 
Table 2. Differentially abundant protein (DAP) spots and their identification by MALDI-TOF/TOF-MS in 0.5 (control), 200 (Cu200), 300 (Cu300) or 400 (Cu400) Cu-treated Citrus grandis and C. sinensis leaves.

\begin{tabular}{|c|c|c|c|c|c|c|c|c|c|c|c|c|c|}
\hline \multirow{2}{*}{ Spot No. } & \multirow{2}{*}{ Protein Identity } & \multirow{2}{*}{ Accession No } & \multirow{2}{*}{$\begin{array}{c}\mathrm{Mr}(\mathrm{kDa}) / \mathrm{PI} \\
\text { Exp. }\end{array}$} & \multirow{2}{*}{$\begin{array}{l}\mathrm{Mr}(\mathrm{kDa}) / \mathrm{PI} \\
\text { Theor. }\end{array}$} & \multirow{2}{*}{$\begin{array}{l}\text { Protein } \\
\text { Score }\end{array}$} & \multirow{2}{*}{$\begin{array}{l}\text { Peptide } \\
\text { Ions }\end{array}$} & \multirow{2}{*}{ NMP } & \multicolumn{4}{|c|}{ Ratio } & \multirow{2}{*}{ CS $(\%)$} & \multirow{2}{*}{ Charge } \\
\hline & & & & & & & & $\mathrm{Cu} 0.5$ & Cu200 & Cu300 & Cu400 & & \\
\hline \multicolumn{14}{|c|}{ Citrus Grandis } \\
\hline \multicolumn{14}{|c|}{ Photosynthesis, Carbohydrate and Energy Metabolism } \\
\hline G1 & $\begin{array}{l}29 \mathrm{kDa} \text { ribonucleoprotein A, chloroplastic; } \\
\text { Ribonucleoprotein At2g37220, chloroplastic }\end{array}$ & Cs6g11900.1 & $30.37 / 5.17$ & $36.20 / 4.33$ & 358 & 186 & 9 & $1.00 \pm 0.11 \mathrm{c}$ & $1.68 \pm 0.19 a b$ & $1.20 \pm 0.14 \mathrm{bc}$ & $2.24 \pm 0.26 \mathrm{a}$ & 8 & 1 \\
\hline G30 & $\begin{array}{l}\text { Photosystem II stability/assembly factor HCF136, } \\
\text { chloroplast, putative }\end{array}$ & Cs7g13970.1 & $45.06 / 8.46$ & $48.11 / 5.91$ & 902 & 42 & 22 & $1.00 \pm 0.11 \mathrm{~b}$ & $1.19 \pm 0.20 \mathrm{~b}$ & $1.17 \pm 0.13 \mathrm{~b}$ & $1.91 \pm 0.12 \mathrm{a}$ & 19 & 1 \\
\hline G31 & $\begin{array}{l}\text { Photosystem II stability/assembly factor HCF136, } \\
\text { chloroplast, putative }\end{array}$ & Cs7g13970.1 & $53.11 / 5.75$ & $59.08 / 5.94$ & 684 & 172 & 28 & $1.00 \pm 0.23 \mathrm{~b}$ & $1.23 \pm 0.25 b$ & $2.40 \pm 0.19 \mathrm{a}$ & $1.58 \pm 0.21 \mathrm{~b}$ & 24 & 1 \\
\hline G10 & $\begin{array}{l}\text { Ferredoxin - NADP reductase, leaf-type isozyme, } \\
\text { chloroplastic }\end{array}$ & Cs1g25510.4 & $55.49 / 5.09$ & $62.32 / 4.92$ & 74 & 47 & 14 & $1.00 \pm 0.11 \mathrm{a}$ & $0.53 \pm 0.08 \mathrm{~b}$ & $0.69 \pm 0.10 \mathrm{ab}$ & $0.36 \pm 0.16 \mathrm{~b}$ & 12 & 1 \\
\hline G42 & $\begin{array}{l}\text { Ferredoxin }- \text { NADP reductase, leaf-type isozyme, } \\
\text { chloroplastic }\end{array}$ & Cs1g25510.4 & $40.48 / 8.68$ & $35.76 / 6.62$ & 458 & 122 & 26 & $1.00 \pm 0.23 \mathrm{~b}$ & $3.66 \pm 0.24 \mathrm{a}$ & $1.64 \pm 0.10 \mathrm{~b}$ & $1.54 \pm 0.32 \mathrm{~b}$ & 23 & 1 \\
\hline G8 & $\begin{array}{c}\text { RuBisCO subunit binding-protein alpha subunit, } \\
\text { chloroplast, putative, expressed; Chaperonin } 60 \\
\text { subunit alpha 1, chloroplastic }\end{array}$ & Cs8g16040.3 & $61.50 / 5.23$ & $68.61 / 4.72$ & 1350 & 169 & 47 & $1.00 \pm 0.22 \mathrm{~b}$ & $0.31 \pm 0.09 \mathrm{c}$ & $1.86 \pm 0.24 \mathrm{a}$ & $1.06 \pm 0.13 b$ & 41 & 1 \\
\hline G9 & $\begin{array}{l}\text { Ribulose bisphosphate carboxylase/oxygenase } \\
\text { activase } 1 \text {, chloroplastic }\end{array}$ & Cs7g31800.3 & $47.86 / 6.29$ & $53.65 / 5.10$ & 617 & 119 & 17 & $1.00 \pm 0.20 \mathrm{ab}$ & $1.38 \pm 0.05 \mathrm{a}$ & $0.80 \pm 0.08 \mathrm{bc}$ & $0.48 \pm 0.08 c$ & 24 & 1 \\
\hline G6 & Sedoheptulose-1,7-bisphosphatase, chloroplastic & Cs7g31640.4 & $42.40 / 5.82$ & $48.51 / 4.64$ & 489 & 84 & 29 & $1.00 \pm 0.22 \mathrm{~b}$ & $1.66 \pm 0.20 \mathrm{a}$ & $1.09 \pm 0.18 \mathrm{ab}$ & $1.32 \pm 0.10 \mathrm{ab}$ & 25 & 1 \\
\hline G38 & $\begin{array}{c}\text { Glyceraldehyde-3-phosphate dehydrogenase B, } \\
\text { chloroplastic }\end{array}$ & Cs3g27520.2 & $48.00 / 7.10$ & $52.45 / 6.38$ & 515 & 104 & 30 & $1.00 \pm 0.27 \mathrm{~b}$ & $2.43 \pm 0.31 \mathrm{a}$ & $1.42 \pm 0.08 \mathrm{~b}$ & $1.34 \pm 0.14 \mathrm{~b}$ & 26 & 1 \\
\hline G29 & Triosephosphate isomerase, cytosolic (Fragment) & Cs5g16495.1 & $26.96 / 5.73$ & $32.66 / 6.15$ & 326 & 129 & 7 & $1.00 \pm 0.04 \mathrm{ab}$ & $1.58 \pm 0.36 \mathrm{a}$ & $0.63 \pm 0.12 b$ & $0.18 \pm 0.10 c$ & 6 & 1 \\
\hline G35 & Triosephosphate isomerase, cytosolic & Cs7g32500.1 & $26.96 / 5.73$ & $31.99 / 6.16$ & 236 & 125 & 14 & $1.00 \pm 0.34 \mathrm{a}$ & $0.69 \pm 0.14 \mathrm{a}$ & $0.51 \pm 0.24 a$ & 0 & 11 & 1 \\
\hline G41 & Triosephosphate isomerase, cytosolic & Cs8g18560.2 & $27.24 / 5.75$ & $33.66 / 6.67$ & 428 & 102 & 17 & $1.00 \pm 0.14 \mathrm{~b}$ & $1.97 \pm 0.27 \mathrm{a}$ & $1.49 \pm 0.05 \mathrm{ab}$ & $1.16 \pm 0.19 \mathrm{~b}$ & 15 & 1 \\
\hline G4 & $\begin{array}{l}\text { Probable 6-phosphogluconolactonase } 4, \\
\text { chloroplastic }\end{array}$ & Orange1.1t02542.1 & $35.38 / 6.24$ & $34.54 / 4.72$ & 1050 & 179 & 25 & $1.00 \pm 0.10 \mathrm{~b}$ & $2.05 \pm 0.35 a$ & $2.04 \pm 0.28 \mathrm{a}$ & $1.30 \pm 0.20 \mathrm{ab}$ & 20 & 1 \\
\hline G36 & Fructose-1,6-bisphosphatase, cytosolic & Cs3g21280.1 & $37.65 / 5.95$ & $47.35 / 6.37$ & 408 & 80 & 22 & $1.00 \pm 0.42 \mathrm{~b}$ & $4.05 \pm 1.70 \mathrm{a}$ & $2.31 \pm 0.11 \mathrm{ab}$ & $1.63 \pm 0.18 \mathrm{ab}$ & 19 & 1 \\
\hline G17 & Malate dehydrogenase [NADP], chloroplastic & Cs7g21820.2 & $47.97 / 6.37$ & $52.65 / 5.59$ & 654 & 104 & 29 & $1.00 \pm 0.28 \mathrm{a}$ & $0.71 \pm 0.05 \mathrm{ab}$ & $1.01 \pm 0.02 \mathrm{a}$ & $0.32 \pm 0.15 b$ & 25 & 1 \\
\hline G37 & Malate dehydrogenase, cytoplasmic & Cs9g10470.1 & $35.54 / 6.10$ & $48.01 / 6.44$ & 212 & 63 & 15 & $1.00 \pm 0.28 \mathrm{~b}$ & $1.66 \pm 0.13 a$ & $1.01 \pm 0.01 \mathrm{~b}$ & $0.82 \pm 0.15 \mathrm{~b}$ & 13 & 1 \\
\hline G39 & Malate dehydrogenase, cytoplasmic & Cs9g10470.1 & $42.35 / 5.94$ & $56.49 / 6.33$ & 193 & 144 & 11 & $1.00 \pm 0.21 \mathrm{~b}$ & $2.76 \pm 0.46 \mathrm{a}$ & $2.11 \pm 0.20 \mathrm{a}$ & $2.35 \pm 0.48 \mathrm{a}$ & 10 & 1 \\
\hline G18 & ATP synthase subunit beta, mitochondrial & Cs2g13550.1 & $59.85 / 6.06$ & $61.56 / 5.45$ & 1240 & 203 & 21 & $1.00 \pm 0.11 b c$ & $0.73 \pm 0.20 c$ & $1.66 \pm 0.12 a$ & $1.40 \pm 0.11 \mathrm{ab}$ & 18 & 1 \\
\hline G23 & ATP synthase gamma chain, chloroplastic & Cs2g03080.1 & $40.62 / 6.08$ & $45.19 / 5.67$ & 594 & 115 & 25 & $1.00 \pm 0.08 \mathrm{bc}$ & $0.64 \pm 0.20 \mathrm{c}$ & $1.65 \pm 0.09 a$ & $1.38 \pm 0.04 \mathrm{ab}$ & 22 & 1 \\
\hline G26 & Bis(5'-adenosy 1)-triphosphatase & Cs9g13060.1 & $17.37 / 5.94$ & $18.09 / 5.99$ & 216 & 105 & 7 & $1.00 \pm 0.07 \mathrm{~b}$ & $1.03 \pm 0.37 \mathrm{~b}$ & $1.47 \pm 0.09 \mathrm{ab}$ & $1.86 \pm 0.14 a$ & 14 & 1 \\
\hline G24 & $\begin{array}{l}\text { Glucose-1-phosphate adenylyltransferase small } \\
\text { subunit 2, chloroplastic }\end{array}$ & Cs2g18800.1 & $57.08 / 6.74$ & $58.03 / 5.66$ & 1120 & 121 & 45 & $1.00 \pm 0.12 \mathrm{~b}$ & $1.97 \pm 0.42 \mathrm{a}$ & $2.24 \pm 0.27 \mathrm{a}$ & $1.64 \pm 0.31 \mathrm{ab}$ & 39 & 1 \\
\hline G25 & $\begin{array}{l}\text { Glucose-1-phosphate adenylyltransferase small } \\
\text { subunit 2, chloroplastic }\end{array}$ & Cs2g18800.1 & $65.86 / 8.50$ & $68.69 / 5.67$ & 751 & 139 & 37 & $1.00 \pm 0.18 \mathrm{~b}$ & $2.11 \pm 0.43 a$ & $1.09 \pm 0.08 \mathrm{~b}$ & $1.36 \pm 0.19 \mathrm{ab}$ & 32 & 1 \\
\hline
\end{tabular}


Table 2. Cont.

\begin{tabular}{|c|c|c|c|c|c|c|c|c|c|c|c|c|c|}
\hline \multirow{2}{*}{ Spot No. } & \multirow{2}{*}{ Protein Identity } & \multirow{2}{*}{ Accession No } & \multirow{2}{*}{$\begin{array}{l}\mathrm{Mr}(\mathrm{kDa}) / \mathrm{PI} \\
\text { Exp. }\end{array}$} & \multirow{2}{*}{$\begin{array}{l}\mathrm{Mr}(\mathrm{kDa}) / \mathrm{PI} \\
\text { Theor. }\end{array}$} & \multirow{2}{*}{$\begin{array}{l}\text { Protein } \\
\text { Score }\end{array}$} & \multirow{2}{*}{$\begin{array}{l}\text { Peptide } \\
\text { Ions }\end{array}$} & \multirow{2}{*}{ NMP } & \multicolumn{4}{|c|}{ Ratio } & \multirow{2}{*}{ CS $(\%)$} & \multirow{2}{*}{ Charge } \\
\hline & & & & & & & & $\mathrm{Cu} 0.5$ & Cu200 & Cu300 & Cu400 & & \\
\hline \multicolumn{14}{|c|}{ Antioxidation and Detoxification } \\
\hline G40 & Glutathione S-transferase & Cs5g32800.1 & $23.83 / 6.17$ & $30.07 / 6.75$ & 389 & 100 & 20 & $1.00 \pm 0.13 \mathrm{~b}$ & $78.9 \pm 28.10 \mathrm{a}$ & $\begin{array}{l}93.20 \pm \\
15.40 \mathrm{a}\end{array}$ & $47.10 \pm 8.39 \mathrm{a}$ & 17 & 1 \\
\hline G34 & Glutathione S-transferase DHAR1, mitochondrial & Cs7g28340.4 & $23.85 / 6.18$ & $30.34 / 6.46$ & 544 & 127 & 21 & $1.00 \pm 0.41 \mathrm{a}$ & $0.57 \pm 0.33 a$ & $0.26 \pm 0.06 \mathrm{a}$ & 0 & 18 & 1 \\
\hline G21 & Copper/zinc superoxide dismutase (Fragment) & Cs3g12000.1 & $15.09 / 5.47$ & $19.86 / 5.80$ & 83 & 46 & 7 & $1.00 \pm 0.07 \mathrm{~b}$ & $1.03 \pm 0.41 \mathrm{~b}$ & $1.18 \pm 0.16 \mathrm{~b}$ & $2.43 \pm 0.51 \mathrm{a}$ & 12 & 1 \\
\hline G33 & Manganese superoxide dismutase (Fragment) & Cs7g29850.1 & $25.29 / 6.79$ & $28.64 / 6.34$ & 520 & 107 & 19 & $1.00 \pm 0.08 \mathrm{~b}$ & $1.51 \pm 0.15 \mathrm{ab}$ & $1.47 \pm 0.16 \mathrm{ab}$ & $2.02 \pm 0.47 \mathrm{a}$ & 17 & 1 \\
\hline G16 & $\begin{array}{l}\text { Quinone oxidoreductase-like protein At1g23740, } \\
\text { chloroplastic }\end{array}$ & Cs7g08640.2 & $41.88 / 8.77$ & $48.90 / 5.38$ & 728 & 189 & 27 & $1.00 \pm 0.12 \mathrm{a}$ & $1.17 \pm 0.08 \mathrm{a}$ & $1.01 \pm 0.04 \mathrm{a}$ & $0.66 \pm 0.03 \mathrm{~b}$ & 23 & 1 \\
\hline \multicolumn{14}{|c|}{ Chaperones and Folding Catalysts } \\
\hline G15 & Probable protein disulfide-isomerase A6 & Cs5g33860.2 & $41.75 / 6.91$ & $44.67 / 5.58$ & 522 & 130 & 28 & $1.00 \pm 0.11 \mathrm{~b}$ & $1.45 \pm 0.18 \mathrm{ab}$ & $1.49 \pm 0.03 \mathrm{ab}$ & $1.60 \pm 0.21 \mathrm{a}$ & 24 & 1 \\
\hline G12 & $20 \mathrm{kDa}$ chaperonin, chloroplastic & Cs4g07030.2 & $26.59 / 8.89$ & $30.93 / 5.32$ & 874 & 186 & 27 & $1.00 \pm 0.18 \mathrm{~b}$ & $0.69 \pm 0.13 \mathrm{~b}$ & $1.66 \pm 0.08 \mathrm{a}$ & $0.73 \pm 0.19 \mathrm{~b}$ & 23 & 1 \\
\hline G11 & Heat shock cognate $70 \mathrm{kDa}$ protein 2 & Cs7g29010.1 & $70.99 / 5.09$ & $74.6 / 4.89$ & 794 & 113 & 5 & $1.00 \pm 0.18 \mathrm{a}$ & $0.81 \pm 0.12 \mathrm{ab}$ & $0.52 \pm 0.12 \mathrm{~b}$ & $0.56 \pm 0.04 \mathrm{~b}$ & 4 & 1 \\
\hline G19 & $\begin{array}{l}\text { Chaperonin CPN60-1, mitochondrial, putative, } \\
\text { expressed }\end{array}$ & Orange1.1t01459.2 & $61.73 / 5.85$ & $68.57 / 5.40$ & 632 & 60 & 46 & $1.00 \pm 0.23 \mathrm{~b}$ & $0.46 \pm 0.04 c$ & $1.76 \pm 0.06 \mathrm{a}$ & $0.81 \pm 0.22 \mathrm{bc}$ & 40 & 1 \\
\hline \multicolumn{14}{|c|}{ Signal Transduction } \\
\hline G3 & Calreticulin-1 & Cs3g15060.3 & $52.52 / 6.29$ & $62.84 / 4.12$ & 122 & 99 & 11 & $1.00 \pm 0.33 \mathrm{a}$ & $0.72 \pm 0.17 \mathrm{ab}$ & $0.54 \pm 0.08 \mathrm{ab}$ & $0.19 \pm 0.02 \mathrm{~b}$ & 15 & 1 \\
\hline G27 & $\begin{array}{l}\text { Major allergen Pru ar } 1 \text { (Major pollen allergen Bet } \mathrm{v} \\
\text { 1-D/H; Major pollen allergen Bet } \mathrm{v} 1-\mathrm{A} \text { ) }\end{array}$ & Cs9g03630.1 & $17.60 / 5.67$ & $21.51 / 6.05$ & 253 & 104 & 19 & $1.00 \pm 0.09 \mathrm{c}$ & $3.02 \pm 0.42 \mathrm{a}$ & $1.66 \pm 0.28 \mathrm{bc}$ & $2.17 \pm 0.49 \mathrm{ab}$ & 17 & 1 \\
\hline G5 & 14-3-3 protein 7 (14-3-3-like protein GF14 epsilon) & Cs3g18200.2 & $28.86 / 4.92$ & $37.48 / 4.61$ & 670 & 174 & 25 & $1.00 \pm 0.20 \mathrm{~b}$ & $1.36 \pm 0.08 \mathrm{ab}$ & $1.39 \pm 0.13 \mathrm{ab}$ & $1.83 \pm 0.16 \mathrm{a}$ & 21 & 1 \\
\hline G22 & Annexin D1 & Cs3g18360.1 & $35.88 / 5.17$ & $46.63 / 5.66$ & 784 & 138 & 31 & $1.00 \pm 0.72 \mathrm{~b}$ & $3.18 \pm 1.21 \mathrm{ab}$ & $4.77 \pm 0.99 \mathrm{a}$ & $2.03 \pm 0.43 \mathrm{ab}$ & 27 & 1 \\
\hline \multicolumn{14}{|c|}{ Cellular Transport } \\
\hline G28 & Ferritin-2, chloroplastic & Cs7g30630.1 & $29.47 / 5.41$ & $32.63 / 5.90$ & 499 & 155 & 27 & $1.00 \pm 0.12 \mathrm{a}$ & $0.55 \pm 0.12 b$ & $0.88 \pm 0.05 \mathrm{ab}$ & $1.21 \pm 0.16 \mathrm{a}$ & 23 & 1 \\
\hline \multicolumn{14}{|c|}{ Cell Wall and Cytoskeleton } \\
\hline G7 & Tubulin beta-6 chain & Cs3g26180.1 & $50.38 / 4.75$ & $62.28 / 4.68$ & 775 & 162 & 30 & $1.00 \pm 0.10 \mathrm{~b}$ & $3.48 \pm 0.55 \mathrm{a}$ & $1.59 \pm 0.08 \mathrm{~b}$ & $1.23 \pm 0.39 \mathrm{~b}$ & 26 & 1 \\
\hline G2 & Endochitinase 1 & Cs8g01850.1 & $35.39 / 4.85$ & $52.52 / 4.16$ & 75 & 33 & 9 & $1.00 \pm 0.16 \mathrm{a}$ & $0.55 \pm 0.27 \mathrm{ab}$ & $0.44 \pm 0.05 \mathrm{~b}$ & $0.75 \pm 0.09 \mathrm{ab}$ & 8 & 1 \\
\hline \multicolumn{14}{|c|}{ Stress Response } \\
\hline G14 & Abscisic stress-ripening protein 1-like & Cs3g21500.1 & $17.88 / 6.00$ & $30.30 / 5.56$ & 323 & 123 & 8 & $1.00 \pm 0.35 b$ & $1.75 \pm 0.48 \mathrm{~b}$ & $1.97 \pm 0.10 \mathrm{~b}$ & $5.75 \pm 0.59 a$ & 29 & 1 \\
\hline \multicolumn{14}{|l|}{ Others } \\
\hline G20 & & Orange1.1t05091.1 & $157.30 / 6.83$ & $19.29 / 5.70$ & 161 & 12 & 31 & $1.00 \pm 0.16 c$ & $1.38 \pm 0.32 \mathrm{bc}$ & $1.80 \pm 0.26 \mathrm{ab}$ & $2.31 \pm 0.19 \mathrm{a}$ & 30 & 1 \\
\hline G32 & $\begin{array}{l}\text { S-adenosyl-L-homocysteine hydrolase } \\
\text { (adenosylhomocysteinase) }\end{array}$ & Orange1.1t01892.1 & $80.71 / 6.26$ & $77.69 / 6.12$ & 577 & 97 & 35 & $1.00 \pm 0.18 \mathrm{~b}$ & $2.72 \pm 0.72 \mathrm{a}$ & $0.87 \pm 0.09 \mathrm{~b}$ & $0.79 \pm 0.10 \mathrm{~b}$ & 3 & 1 \\
\hline \multicolumn{14}{|c|}{ Unidentified Protein Spots } \\
\hline G13 & Receptor serine-threonine protein kinase, putative & Cs9g04750.2 & $25.57 / 8.87$ & $23.24 / 5.56$ & 45 & 109 & 13 & $1.00 \pm 0.15 \mathrm{~b}$ & $1.06 \pm 0.28 \mathrm{~b}$ & $1.13 \pm 0.25 \mathrm{~b}$ & $2.12 \pm 0.35 \mathrm{a}$ & 22 & 1 \\
\hline
\end{tabular}


Table 2. Cont.

\begin{tabular}{|c|c|c|c|c|c|c|c|c|c|c|c|c|c|}
\hline \multirow{2}{*}{ Spot No. } & \multirow{2}{*}{ Protein Identity } & \multirow{2}{*}{ Accession No } & \multirow{2}{*}{$\begin{array}{l}\mathrm{Mr}(\mathrm{kDa}) / \mathrm{PI} \\
\text { Exp. }\end{array}$} & \multirow{2}{*}{$\begin{array}{l}\mathrm{Mr}(\mathrm{kDa}) / \mathrm{PI} \\
\text { Theor. }\end{array}$} & \multirow{2}{*}{$\begin{array}{l}\text { Protein } \\
\text { Score }\end{array}$} & \multirow{2}{*}{$\begin{array}{l}\text { Peptide } \\
\text { Ions }\end{array}$} & \multirow{2}{*}{ NMP } & \multicolumn{4}{|c|}{ Ratio } & \multirow{2}{*}{ CS $(\%)$} & \multirow{2}{*}{ Charge } \\
\hline & & & & & & & & $\mathrm{Cu} 0.5$ & Cu200 & Cu300 & Cu400 & & \\
\hline \multicolumn{14}{|c|}{ Citrus Sinensis } \\
\hline \multicolumn{14}{|c|}{ Photosynthesis, Carbohydrate and Energy Metabolism } \\
\hline S19 & Chlorophyll a-b binding protein 8,chloroplastic & Cs3g06180.2 & $29.52 / 6.84$ & $32.89 / 5.42$ & 222 & 95 & 12 & $1.00 \pm 0.25 b$ & $1.80 \pm 0.27 \mathrm{a}$ & $1.28 \pm 0.05 \mathrm{ab}$ & $0.44 \pm 0.09 \mathrm{c}$ & 10 & 1 \\
\hline S41 & Protease Do-like 1, chloroplastic & Cs2g28080.1 & $53.11 / 5.75$ & $95.23 / 4.75$ & 553 & 139 & 17 & $1.00 \pm 0.15 \mathrm{a}$ & $0.12 \pm 0.05 \mathrm{c}$ & $0.68 \pm 0.04 \mathrm{ab}$ & $0.51 \pm 0.10 \mathrm{bc}$ & 15 & 1 \\
\hline S13 & PsbP domain-containing protein 3 , chloroplastic & Cs3g27720.1 & $27.63 / 8.28$ & $21.66 / 5.68$ & 366 & 126 & 9 & $1.00 \pm 0.17 \mathrm{a}$ & $0.81 \pm 0.11 \mathrm{ab}$ & $0.55 \pm 0.10 \mathrm{bc}$ & $0.36 \pm 0.11 \mathrm{c}$ & 12 & 1 \\
\hline S2 & $\begin{array}{l}29 \mathrm{kDa} \text { ribonucleoprotein A, chloroplastic; } \\
\text { Ribonucleoprotein At2g37220, chloroplastic }\end{array}$ & Cs6g11900.1 & $30.37 / 5.17$ & $45.1 / 6.21$ & 418 & 195 & 13 & $1.00 \pm 0.08 \mathrm{a}$ & $0.82 \pm 0.16 \mathrm{ab}$ & $0.43 \pm 0.09 \mathrm{c}$ & $0.53 \pm 0.06 \mathrm{bc}$ & 11 & 1 \\
\hline S32 & $\begin{array}{l}29 \mathrm{kDa} \text { ribonucleoprotein A, chloroplastic; } \\
\text { Ribonucleoprotein At2g37220, chloroplastic }\end{array}$ & Cs7g01430.1 & $28.53 / 7.78$ & $33.75 / 5.11$ & 392 & 114 & 19 & $1.00 \pm 0.20 \mathrm{a}$ & $0.76 \pm 0.02 \mathrm{ab}$ & $0.49 \pm 0.14 \mathrm{~b}$ & 0 & 23 & 1 \\
\hline S17 & Oxygen-evolving enhancer protein 1 , chloroplastic & Cs1g23450.1 & $35.38 / 5.83$ & $24.74 / 5.56$ & 261 & 116 & 7 & $1.00 \pm 0.05 c$ & $1.06 \pm 0.01 \mathrm{bc}$ & $1.34 \pm 0.10 \mathrm{ab}$ & $1.54 \pm 0.14 \mathrm{a}$ & 19 & 1 \\
\hline S3 & Carbonic anhydrase, chloroplastic & Cs2g28060.4 & $36.77 / 6.66$ & $53.09 / 6.25$ & 171 & 162 & 5 & $1.00 \pm 0.26 c$ & $3.02 \pm 0.38 \mathrm{ab}$ & $3.14 \pm 0.23 \mathrm{a}$ & $1.18 \pm 0.35 \mathrm{bc}$ & 5 & 1 \\
\hline S11 & $\begin{array}{l}\text { Rubisco subunit binding-protein alpha subunit, } \\
\text { chloroplast, putative, expressed; Chaperonin } 60 \\
\text { subunit alpha 1, chloroplastic } \\
\end{array}$ & Cs8g16040.1 & $61.50 / 5.23$ & 99.02/5.94 & 1250 & 182 & 39 & $1.00 \pm 0.1 \mathrm{a}$ & $0.70 \pm 0.16 \mathrm{a}$ & $0.80 \pm 0.17 \mathrm{a}$ & $0.27 \pm 0.04 \mathrm{~b}$ & 34 & 1 \\
\hline S9 & $\begin{array}{l}\text { Ribulose bisphosphate carboxylase/oxygenase } \\
\text { activase } 1 \text {, chloroplastic }\end{array}$ & Cs7g31800.3 & $50.90 / 5.33$ & $81.78 / 6.10$ & 505 & 107 & 21 & $1.00 \pm 0.23 \mathrm{a}$ & $0.75 \pm 0.10 \mathrm{ab}$ & $0.66 \pm 0.10 \mathrm{ab}$ & $0.51 \pm 0.04 \mathrm{~b}$ & 28 & 1 \\
\hline S14 & $\begin{array}{l}\text { Ribulose bisphosphate carboxylase/oxygenase } \\
\text { activase } 1 \text {, chloroplastic }\end{array}$ & Cs7g31800.3 & $46.96 / 5.94$ & $75.7 / 5.65$ & 505 & 107 & 21 & $1.00 \pm 0.11 \mathrm{a}$ & $0.65 \pm 0.02 \mathrm{~b}$ & $0.48 \pm 0.05 b$ & $0.45 \pm 0.05 \mathrm{~b}$ & 28 & 1 \\
\hline S4 & $\begin{array}{l}\text { Ribulose bisphosphate carboxylase/oxygenase } \\
\text { activase } 1 \text {, chloroplastic }\end{array}$ & Cs7g31800.3 & $46.96 / 5.94$ & $83.26 / 6.21$ & 579 & 139 & 19 & $1.00 \pm 0.46 \mathrm{a}$ & 0 & $0.38 \pm 0.03 a$ & $0.77 \pm 0.06 \mathrm{a}$ & 17 & 1 \\
\hline S10 & $\begin{array}{l}\text { Ribulose bisphosphate carboxylase/oxygenase } \\
\text { activase } 1 \text {, chloroplastic }\end{array}$ & Cs7g31800.3 & $46.96 / 5.94$ & $79.78 / 6.02$ & 641 & 175 & 24 & $1.00 \pm 0.16 \mathrm{~b}$ & $1.38 \pm 0.30 \mathrm{~b}$ & $2.16 \pm 0.23 \mathrm{a}$ & $1.05 \pm 0.04 \mathrm{~b}$ & 21 & 1 \\
\hline S21 & Phosphoribulokinase, chloroplastic & Cs3g08480.1 & $45.19 / 5.97$ & $67.41 / 5.58$ & 686 & 137 & 31 & $1.00 \pm 0.05 \mathrm{a}$ & $1.01 \pm 0.04 \mathrm{a}$ & $1.02 \pm 0.15 \mathrm{a}$ & 0 & 27 & 1 \\
\hline S33 & Sedoheptulose-1,7-bisphosphatase, chloroplastic & Cs7g31640.4 & $36.77 / 6.66$ & $43.56 / 4.96$ & 576 & 101 & 27 & $1.00 \pm 0.06 \mathrm{a}$ & $1.02 \pm 0.06 \mathrm{a}$ & $0.75 \pm 0.24 \mathrm{a}$ & 0 & 23 & 1 \\
\hline S44 & Malate dehydrogenase, mitochondrial & Cs7g25390.1 & $35.48 / 8.52$ & $66.81 / 4.32$ & 613 & 144 & 24 & $1.00 \pm 0.02 \mathrm{~b}$ & $1.18 \pm 0.21 \mathrm{ab}$ & $0.95 \pm 0.15 b$ & $1.64 \pm 0.26 \mathrm{a}$ & 21 & 1 \\
\hline S45 & Malate dehydrogenase, mitochondrial & Cs7g25390.3 & $37.65 / 5.95$ & $63.69 / 4.53$ & 287 & 171 & 9 & $1.00 \pm 0.16 \mathrm{bc}$ & $1.15 \pm 0.2 \mathrm{~b}$ & $0.46 \pm 0.21 \mathrm{c}$ & $1.85 \pm 0.22 \mathrm{a}$ & 8 & 1 \\
\hline S30 & Enolase & Cs6g15540.1 & $15.09 / 5.47$ & $18.14 / 4.99$ & 928 & 154 & 30 & $1.00 \pm 0.12 \mathrm{a}$ & $0.53 \pm 0.07 \mathrm{~b}$ & $0.63 \pm 0.09 \mathrm{~b}$ & $0.22 \pm 0.08 \mathrm{c}$ & 26 & 1 \\
\hline S36 & Enolase & Cs6g15540.1 & $47.79 / 5.54$ & $89.59 / 5.04$ & 531 & 136 & 15 & $1.00 \pm 0.25 \mathrm{~b}$ & $1.04 \pm 0.22 \mathrm{~b}$ & $1.78 \pm 0.13 a$ & $1.92 \pm 0.11 \mathrm{a}$ & 47 & 1 \\
\hline S43 & $\begin{array}{c}\text { Dihydrolipoyllysine-residue succinyltransferase } \\
\text { component of 2-oxoglutarate dehydrogenase } \\
\text { complex 2, mitochondrial }\end{array}$ & Cs2g21190.3 & $40.39 / 6.95$ & $87.84 / 4.79$ & 268 & 144 & 8 & $1.00 \pm 0.26 \mathrm{a}$ & $0.56 \pm 0.20 \mathrm{ab}$ & $0.30 \pm 0.06 \mathrm{~b}$ & 0 & 25 & 1 \\
\hline \multicolumn{14}{|c|}{ Antioxidation and Detoxification } \\
\hline S1 & 2-Cys peroxiredoxin BAS1, chloroplastic & Cs6g13880.1 & $29.49 / 7.65$ & $30.56 / 6.56$ & 465 & 161 & 17 & $1.00 \pm 0.10 \mathrm{a}$ & $0.89 \pm 0.11 \mathrm{a}$ & 0 & $0.81 \pm 0.19 \mathrm{a}$ & 15 & 1 \\
\hline S20 & Cysteine synthase, chloroplastic/chromoplastic & Orange1.1t02144.1 & $41.35 / 8.29$ & $56.87 / 5.55$ & 880 & 197 & 28 & $1.00 \pm 0.14 \mathrm{a}$ & 0 & $0.91 \pm 0.09 \mathrm{a}$ & $1.17 \pm 0.06 \mathrm{a}$ & 21 & 1 \\
\hline S39 & Cysteine synthase & Cs9g06970.1 & $29.29 / 6.78$ & $60.56 / 4.69$ & 114 & 49 & 6 & $1.00 \pm 0.28 \mathrm{a}$ & $1.04 \pm 0.12 \mathrm{a}$ & $0.78 \pm 0.15 \mathrm{a}$ & 0 & 17 & 1 \\
\hline S34 & L-ascorbate peroxidase 1 , cytosolic & Cs8g17370.1 & $28.68 / 5.42$ & $46.45 / 5.05$ & 392 & 114 & 19 & $1.00 \pm 0.05 a$ & $0.82 \pm 0.18 \mathrm{a}$ & $0.77 \pm 0.14 a$ & $0.35 \pm 0.01 \mathrm{~b}$ & 23 & 1 \\
\hline S24 & Glutathione peroxidase (Fragment) & Cs5g03830.1 & $18.58 / 5.72$ & $25.08 / 5.15$ & 646 & 135 & 21 & $1.00 \pm 0.14 \mathrm{a}$ & $1.02 \pm 0.07 \mathrm{a}$ & $0.88 \pm 0.16 \mathrm{ab}$ & $0.55 \pm 0.12 b$ & 30 & 1 \\
\hline
\end{tabular}


Table 2. Cont.

\begin{tabular}{|c|c|c|c|c|c|c|c|c|c|c|c|c|c|}
\hline \multirow{2}{*}{ Spot No. } & \multirow{2}{*}{ Protein Identity } & \multirow{2}{*}{ Accession No } & \multirow{2}{*}{$\begin{array}{l}\mathrm{Mr}(\mathrm{kDa}) / \mathrm{PI} \\
\text { Exp. }\end{array}$} & \multirow{2}{*}{$\begin{array}{l}\mathrm{Mr}(\mathrm{kDa}) / \mathrm{PI} \\
\text { Theor. }\end{array}$} & \multirow{2}{*}{$\begin{array}{l}\text { Protein } \\
\text { Score }\end{array}$} & \multirow{2}{*}{$\begin{array}{l}\text { Peptide } \\
\text { Ions }\end{array}$} & \multirow[b]{2}{*}{ NMP } & \multicolumn{4}{|c|}{ Ratio } & \multirow{2}{*}{ CS $(\%)$} & \multirow{2}{*}{ Charge } \\
\hline & & & & & & & & $\mathrm{Cu} 0.5$ & Cu200 & Cu300 & Cu400 & & \\
\hline \multicolumn{14}{|c|}{ Chaperones and Folding Catalysts } \\
\hline S16 & Luminal-binding protein 5 & Cs5g01840.2 & $73.56 / 5.09$ & $108.09 / 5.72$ & 578 & 112 & 27 & $1.00 \pm 0.18 \mathrm{~b}$ & $1.07 \pm 0.21 \mathrm{~b}$ & $2.57 \pm 0.19 a$ & $2.39 \pm 0.23 a$ & 23 & 1 \\
\hline S8 & $\begin{array}{l}\text { Peptidyl-prolylcis-transisomerase CYP37, } \\
\text { chloroplastic }\end{array}$ & Cs1g06710.1 & $50.39 / 6.42$ & $58.95 / 5.92$ & 109 & 92 & 5 & $1.00 \pm 0.13 \mathrm{a}$ & $1.03 \pm 0.07 \mathrm{a}$ & $0.73 \pm 0.05 \mathrm{ab}$ & $0.66 \pm 0.12 b$ & 2 & 1 \\
\hline S26 & $\begin{array}{l}\text { Chaperonin CPN60-1, mitochondrial, putative, } \\
\text { expressed }\end{array}$ & Orange1.1t01459.2 & $46.12 / 8.24$ & $52.38 / 5.28$ & 727 & 152 & 39 & $1.00 \pm 0.13 \mathrm{a}$ & $0.90 \pm 0.14 \mathrm{a}$ & $1.16 \pm 0.12 \mathrm{a}$ & 0 & 34 & 1 \\
\hline \multicolumn{14}{|c|}{ Signal Transduction } \\
\hline S35 & $\begin{array}{l}\text { Major allergen Pru ar } 1 \text { (Major pollen allergen Bet } \mathrm{v} \\
\text { 1-D/H; Major pollen allergen Bet } \mathrm{v} 1-\mathrm{A})\end{array}$ & Cs9g03630.1 & $48.33 / 6.19$ & $89.59 / 5.09$ & 230 & 94 & 15 & $1.00 \pm 0.07 \mathrm{a}$ & $0.57 \pm 0.15 b$ & $1.20 \pm 0.13 \mathrm{a}$ & $0.91 \pm 0.05 \mathrm{ab}$ & 13 & 1 \\
\hline S7 & 14-3-3 protein 6 & Orange1.1t01991.1 & $29.44 / 4.84$ & $45.11 / 6.09$ & 439 & 137 & 18 & $1.00 \pm 0.18 \mathrm{a}$ & $0.28 \pm 0.02 b$ & $0.51 \pm 0.13 \mathrm{ab}$ & $0.54 \pm 0.04 \mathrm{ab}$ & 16 & 1 \\
\hline \multicolumn{14}{|c|}{ Cellular Transport } \\
\hline S5 & Ferritin-3, chloroplastic & Cs6g09150.2 & $28.97 / 5.46$ & $41.03 / 5.97$ & 406 & 129 & 13 & $1.00 \pm 0.03 a$ & $0.65 \pm 0.03 \mathrm{~b}$ & $0.61 \pm 0.12 \mathrm{~b}$ & $0.46 \pm 0.03 \mathrm{~b}$ & 33 & 1 \\
\hline \multicolumn{14}{|c|}{ Nucleic acid Metabolism } \\
\hline S42 & RuvB-like helicase 1 & Cs6g16920.1 & $38.08 / 6.90$ & $90.61 / 4.73$ & 268 & 99 & 16 & $1.00 \pm 0.17 \mathrm{a}$ & $0.82 \pm 0.33 \mathrm{a}$ & $0.56 \pm 0.08 \mathrm{a}$ & 0 & 25 & 1 \\
\hline \multicolumn{14}{|l|}{ Others } \\
\hline S27 & & Orange1.1t05091.1 & $53.64 / 5.26$ & $85.72 / 5.32$ & 246 & 73 & 19 & $1.00 \pm 0.38 \mathrm{a}$ & $0.15 \pm 0.01 \mathrm{~b}$ & $0.23 \pm 0.04 \mathrm{~b}$ & $0.44 \pm 0.18 \mathrm{ab}$ & 17 & 1 \\
\hline S28 & & Orange1.1t05091.1 & $61.73 / 5.85$ & $95.17 / 5.28$ & 212 & 70 & 16 & $1.00 \pm 0.24 \mathrm{a}$ & $0.91 \pm 0.15 \mathrm{a}$ & $0.32 \pm 0.05 b$ & $0.60 \pm 0.07 \mathrm{ab}$ & 14 & 1 \\
\hline S31 & & Orange1.1t05091.1 & $177.77 / 7.11$ & $24.86 / 4.86$ & 170 & 45 & 15 & $1.00 \pm 0.07 \mathrm{a}$ & $0.75 \pm 0.15 \mathrm{ab}$ & $0.53 \pm 0.12 b$ & 0 & 14 & 1 \\
\hline S23 & Anthranilate N-methyltransferase & Cs5g24940.1 & $39.48 / 5.20$ & $23.56 / 5.23$ & 300 & 146 & 17 & $1.00 \pm 0.03 a$ & $0.93 \pm 0.02 \mathrm{a}$ & $0.27 \pm 0.10 \mathrm{~b}$ & 0 & 15 & 1 \\
\hline S37 & $\begin{array}{l}\text { S-adenosyl-L-homocysteine hydrolase } \\
\text { (adenosylhomocysteinase) }\end{array}$ & Orange1.1t01892.1 & $17.60 / 5.67$ & $26.91 / 4.83$ & 776 & 172 & 24 & $1.00 \pm 0.12 \mathrm{a}$ & $1.18 \pm 0.07 \mathrm{a}$ & $0.73 \pm 0.24 \mathrm{a}$ & 0 & 21 & 1 \\
\hline S38 & Dihydroflavonol-4-reductase & Cs3g01140.1 & $15.15 / 4.94$ & $43.76 / 4.84$ & 396 & 152 & 14 & $1.00 \pm 0.07 \mathrm{a}$ & $1.28 \pm 0.35 \mathrm{a}$ & $2.04 \pm 0.45 \mathrm{a}$ & 0 & 12 & 1 \\
\hline \multicolumn{14}{|c|}{ Unidentified Protein Spots } \\
\hline S6 & $\begin{array}{l}\text { Light-harvesting chlorophyll-a/b binding protein } \\
\text { Lhca6 (Fragment) }\end{array}$ & Cs7g27290.1 & $26.56 / 5.43$ & $35.60 / 5.91$ & 105 & 50 & 4 & $1.00 \pm 0.24 \mathrm{a}$ & $0.87 \pm 0.06 \mathrm{ab}$ & $0.71 \pm 0.09 \mathrm{ab}$ & $0.49 \pm 0.02 \mathrm{~b}$ & 31 & 1 \\
\hline S12 & Thioredoxin M-type, chloroplastic & Cs3g20630.1 & $19.91 / 8.83$ & $16.79 / 5.85$ & 60 & 43 & 4 & $1.00 \pm 0.18 \mathrm{a}$ & $0.95 \pm 0.13 \mathrm{a}$ & $0.80 \pm 0.08 \mathrm{ab}$ & $0.40 \pm 0.17 \mathrm{~b}$ & 7 & 1 \\
\hline S15 & $\begin{array}{l}\text { Nicotinate-nucleotide pyrophosphorylase } \\
\text { [carboxylatingy, putative }\end{array}$ & Orange1.1t04780.1 & $55.49 / 5.09$ & $91.64 / 5.82$ & 50 & 131 & 26 & $1.00 \pm 0.26 \mathrm{a}$ & $0.79 \pm 0.18 \mathrm{ab}$ & $0.59 \pm 0.04 \mathrm{ab}$ & $0.30 \pm 0.02 \mathrm{~b}$ & 58 & 1 \\
\hline S18 & Disease resistance protein RFL1, putative & Cs3g08210.1 & $49.77 / 9.44$ & $31.35 / 5.42$ & 50 & 18 & 10 & $1.00 \pm 0.20 \mathrm{a}$ & $0.39 \pm 0.13 \mathrm{~b}$ & $0.33 \pm 0.04 \mathrm{~b}$ & $0.19 \pm 0.1 \mathrm{~b}$ & 17 & 1 \\
\hline S22 & $\begin{array}{c}\text { Dehydration-responsive family protein, putative, } \\
\text { expressed }\end{array}$ & Orange1.1t00308.3 & $49.93 / 5.04$ & $88.43 / 5.57$ & 57 & 117 & 24 & $1.00 \pm 0.19 a$ & $0.94 \pm 0.06 \mathrm{ab}$ & $0.87 \pm 0.02 \mathrm{ab}$ & $0.65 \pm 0.07 \mathrm{~b}$ & 57 & 1 \\
\hline S25 & $\begin{array}{c}\text { Transducin/WD40 domain-containing protein-like } \\
\text { protein }\end{array}$ & Cs9g09840.1 & $29.76 / 6.18$ & $31.98 / 5.16$ & 67 & 41 & 9 & $1.00 \pm 0.14 a$ & $0.83 \pm 0.15 a$ & $0.51 \pm 0.12 \mathrm{a}$ & 0 & 8 & 1 \\
\hline S29 & ATPase 8, plasma membrane-type & Cs4g01370.1 & $14.72 / 5.41$ & $22.10 / 5.07$ & 68 & 98 & 11 & $1.00 \pm 0.09 \mathrm{a}$ & $0.90 \pm 0.07 \mathrm{ab}$ & $0.72 \pm 0.05 \mathrm{bc}$ & $0.50 \pm 0.09 \mathrm{c}$ & 21 & 1 \\
\hline S40 & $\begin{array}{l}\text { 4-hydroxy-3-methylbut-2-enyl diphosphate } \\
\text { reductase }\end{array}$ & Cs5g28200.1 & $52.22 / 6.60$ & $81.69 / 4.72$ & 63 & 49 & 5 & $1.00 \pm 0.16 \mathrm{a}$ & $0.68 \pm 0.06 \mathrm{a}$ & $0.66 \pm 0.14 \mathrm{a}$ & 0 & 17 & 1 \\
\hline
\end{tabular}

Note: Spot number corresponds to the 2-DE imagines in Figure 2. Ratio means the ratio of 0.5, 200, 300 or $400 \mu \mathrm{M} \mathrm{Cu}$-treated leaves to $0.5 \mu \mathrm{M}$ Cu-treated leaves. NMP: the number of matched peptides; CS: covered sequence. Means $( \pm \mathrm{SE}, n=3)$ with a row followed by different letters are significant different at $p<0.05$. 
DAPs were mainly involved in photosynthesis, carbohydrate and energy metabolism, antioxidation and detoxification, protein folding and assembly (viz., chaperones and folding catalysts), and others. Cell wall, cytoskeleton (G7 and G2), and stress response (G14) related DAPs were obtained only in Cu-treated C. grandis leaves, but nucleic acid metabolism related DAP (S42) was identified only in Cu-treated C. sinensis leaves (Table 2 and Figure 3).

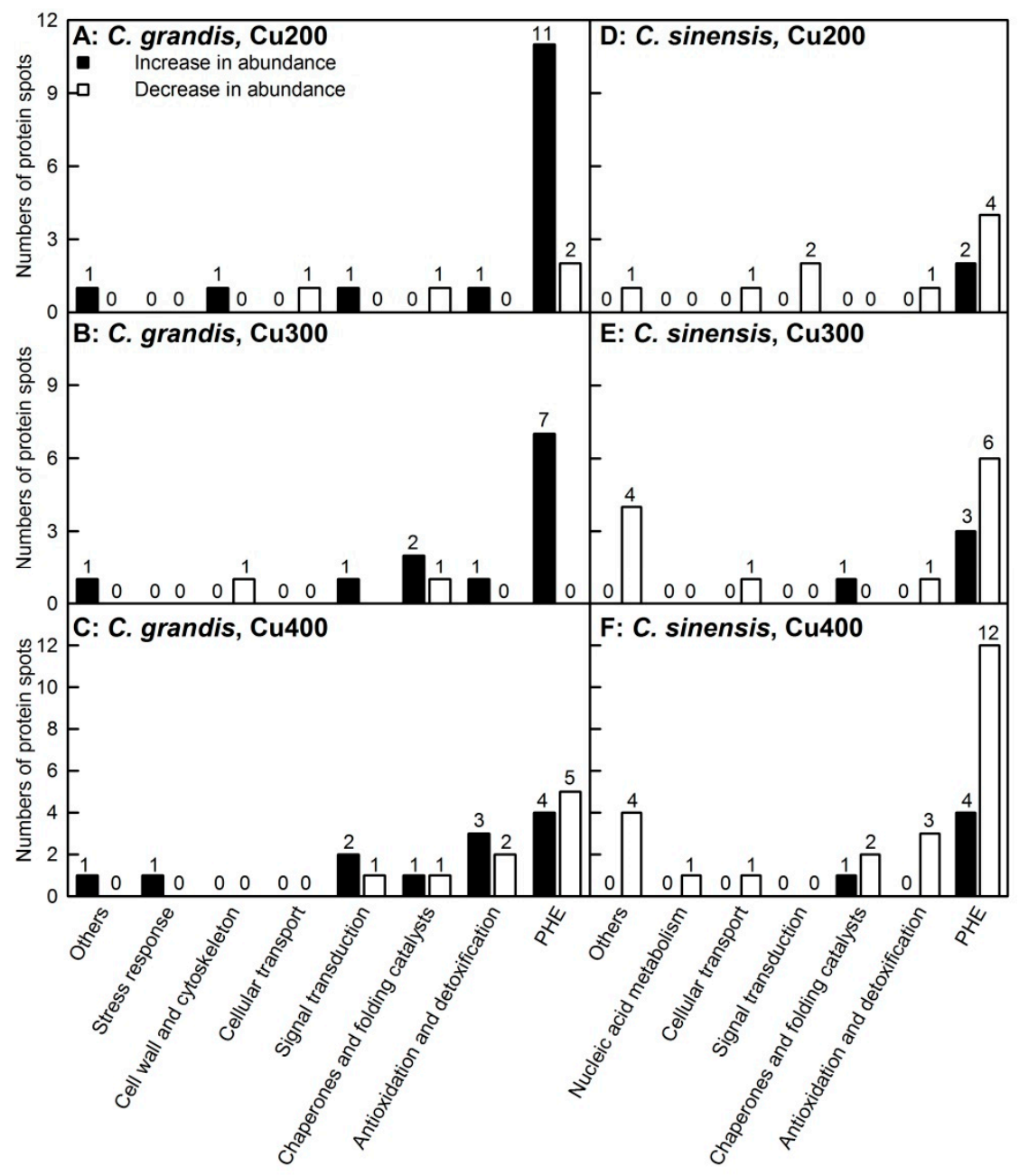

Figure 3. Differentially abundant proteins (DAPs) in 200, 300 and $400 \mu \mathrm{M}$ Cu-treated Citrus grandis (A-C) and Citrus sinensis (D-F) leaves. PHE: photosynthesis, carbohydrate and energy metabolism.

\subsection{KEGG Pathway Analysis of DAPs}

For total DAPs in C. grandis leaves, there were eight significantly enriched KEGG pathways-namely carbon fixation in photosynthetic organisms (ko00710), exosome (ko04147), glycolysis/gluconeogenesis (ko00010), fructose and mannose metabolism (ko00051), photosynthesis (ko00195), chaperones and folding catalysts (ko03110), photosynthesis proteins (ko00194) and inositol phosphate metabolism (ko00562). Four, six, and ten KEGG pathways were significantly enriched by DAPs in 200, 300, and $400 \mu \mathrm{M} \mathrm{Cu}$-treated C. grandis leaves, respectively. For total DAPs in C. sinensis leaves, carbon fixation in photosynthetic organisms, photosynthesis proteins, exosome, tricarboxylic acid (TCA) cycle (ko00020) and photosynthesis were the significantly enriched KEGG pathways. One [photosynthesis-antenna proteins (ko00196)], one (exosome) and five KEGG pathways were significantly enriched by DAPs in 200, 300 and $400 \mu \mathrm{M}$ Cu-treated C. sinensis leaves, respectively (Supplementary Figure S6). 


\subsection{PCA Loading Plots and Correlation Matrices of DAPs}

As shown in Figure 4 and Supplementary Tables S2 and S3, PC1 and PC2 accounted for 30.5\% and $26.5 \%$, and $45.8 \%$ and $17.4 \%$ of the total variation in C. grandis and C. sinensis leaves, respectively. The association patterns of DAPs were more obvious in C. sinensis leaves than those in C. grandis leaves. Similarly, more positive and negative relationships between DAP spots existed in $C$. sinensis leaves than those in C. grandis leaves (Supplementary Figure S7).
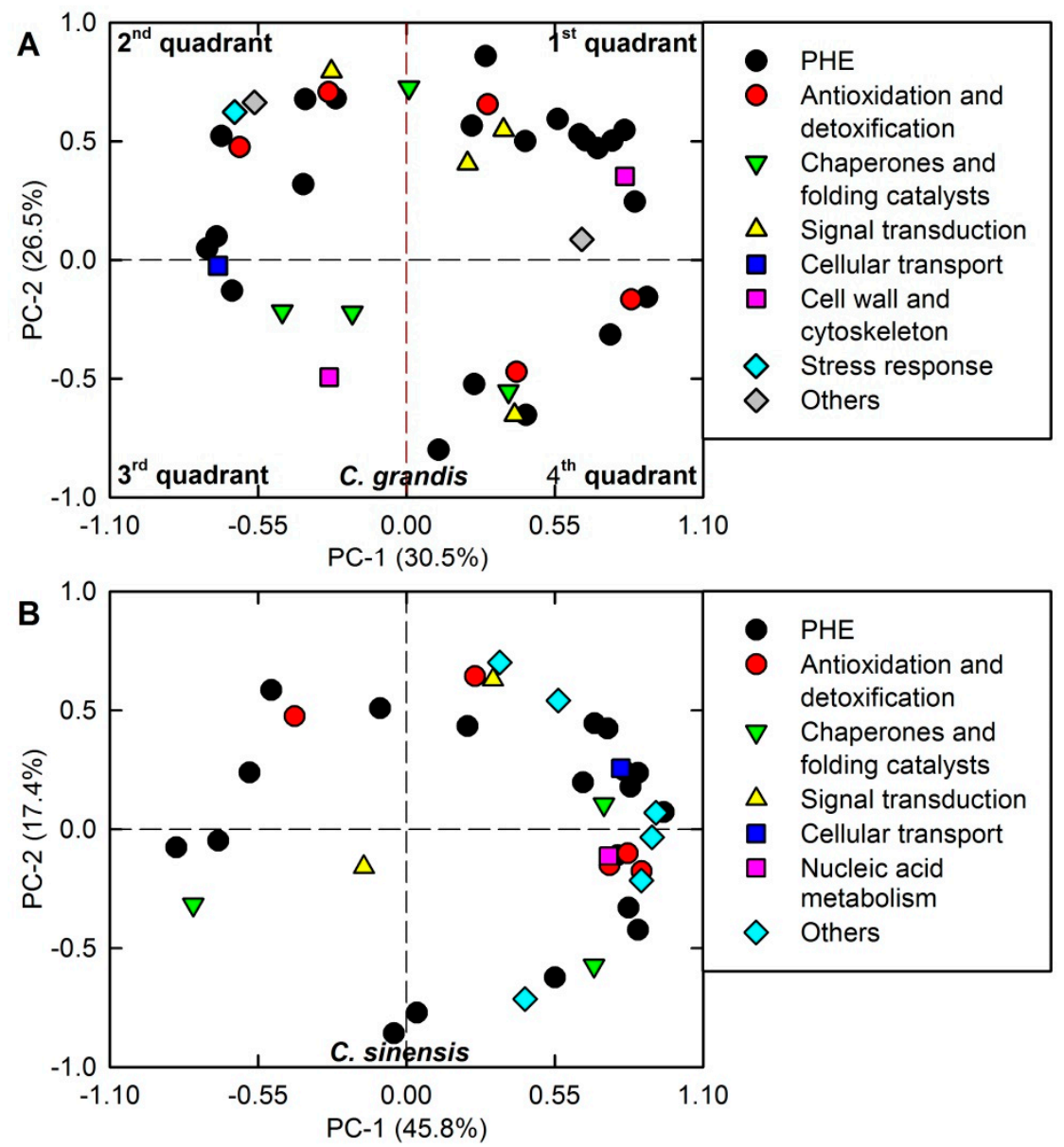

Figure 4. Principal component analysis (PCA) of differentially abundant proteins (DAPs) in Cu-treated Citrus grandis (A) and Citrus sinensis (B) leaves. PHE: photosynthesis, carbohydrate and energy metabolism.

\section{5. qRT-PCR Analysis of Genes for DAPs}

The expression levels of genes for 22 DAPs from $400 \mu \mathrm{M}$ Cu-treated C. grandis (viz., G3, G9, G10, G11, G14, G26, G29, G33, G34, and G35) and C. sinensis (viz., S2, S5, S9, S16, S17, S23, S24, S30, S32, S33, S37, and S43) leaves were analyzed by qRT-PCR. With the exceptions of G26, G33, S9, S16, S23, S32, and S33, the abundances of the other 16 DAPs matched well with the expression levels of the corresponding genes regardless of whether PRPF31 or actin served as an internal standard (Table 2 and Supplementary Figure S8).

\section{Discussion}

\subsection{DAPs Related to Photosynthesis, Carbohydrate and Energy Metabolism}

Excess $\mathrm{Cu}$-treated $C$. grandis and C. sinensis leaves had lower $\mathrm{CO}_{2}$ assimilation (Figure 1) and higher concentrations of nonstructural carbohydrates relative to controls [5]. Accordingly, many 
Cu-toxicity-responsive proteins related to photosynthesis, carbohydrate and energy were identified in these leaves (Table 2 and Figure 3). Damkjær et al. reported that Arabidopsis mutants lacking light-harvesting chlorophyll (Chl) a/b binding protein $L h c b 3$ had a lower maximum photosystem (PSII) efficiency of dark-adapted leaves $\left(\mathrm{F}_{\mathrm{v}} / \mathrm{F}_{\mathrm{m}}\right)$ than wild type under high light condition and still displayed a lower $\mathrm{F}_{\mathrm{v}} / \mathrm{F}_{\mathrm{m}}$ after $7 \mathrm{~d}$ of recovery under normal light, implying that PSII in these plants suffered from photoinhibition under high light [31]. The abundance of Chl a-b binding protein 8 (Lhca3; S19) was increased and decreased in 200 and $400 \mu \mathrm{M}$ Cu-treated C. sinensis leaves, respectively. Thus, the decreased abundance of Lhca3 in $400 \mu \mathrm{M}$ Cu-treated C. sinensis leaves might contribute to the $\mathrm{Cu}$-induced photoinhibition. This could explain why photoinhibition was slightly greater in $400 \mu \mathrm{M}$ $\mathrm{Cu}$-treated C. sinensis leaves than that in $400 \mu \mathrm{M} \mathrm{Cu}$-treated C. grandis leaves [5]. Also, the abundance of protease Do-like 1 (DEGP1; S41) was decreased in 200 and $400 \mu \mathrm{M}$ Cu-treated C. sinensis leaves. DEGP1, an enzyme responsible for the degradation of damaged proteins, plays a role in photoinhibition repair of PSII in Arabidopsis [32]. Also, the abundance of PsbP domain-containing protein 3 (PPD3, S13) involved in PSII light reaction was decreased in 300 and $400 \mu \mathrm{M}$ Cu-treated C. sinensis leaves.

Phosphorylation of PSII antenna protein RNA-binding protein CP29, localized in chloroplasts, was induced under conditions of decreased photosynthetic capacity and excess light. Maize plants lacking the ability to perform the phosphorylation of CP29 were more sensitive to cold-induced photoinhibition [33]. CP29 phosphorylation has been indicated to play a role in lowering ${ }^{1} \mathrm{O}_{2}$ generation and improving excess energy dissipation [34]. The abundance of CP29A (G1) was increased in 200 and $400 \mu \mathrm{M}$ Cu-treated C. grandis leaves, while the abundances of CP29A (S2 and S32) were decreased in 300 and $400 \mu \mathrm{M}$ Cu-treated C. sinensis leaves. The different response of CP20A to excess $\mathrm{Cu}$ between the two agreed with the report that excess $\mathrm{Cu}$ had less influence on $\mathrm{Chl}$ a fluorescence (OJIP) transients in C. grandis leaves than those in C. sinensis leaves [5]. Similarly, the abundance of PSII stability/assembly factor HCF136, an essential protein for the stability/assembly of PSII, was increased in 300 (G31) and 400 (G30) $\mu \mathrm{M}$ Cu-treated C. grandis leaves, but not in Cu-treated C. sinensis leaves. Increased abundance of HCF136 has been obtained in cadmium (Cd) treated Arabidopsis shoots [35]. However, the abundances of oxygen-evolving enhancer protein 1 (PSBO2, S17) were enhanced significantly in $400 \mu \mathrm{M}$ Cu-treated C. sinensis leaves. PSBO2 is required for the stability of the photosynthetic water-splitting complex [36]. Interestingly, the damage of the oxygen evolving complexes (OEC) was greater in C. sinensis leaves than that in C. grandis leaves under $400 \mu \mathrm{M} \mathrm{Cu}$ [5]. Evidently, other factors play a role in stabilizing the water-splitting complex.

The abundance of G10 (ferredoxin-NADP reductase, leaf-type isozyme (LFNR2)) was decreased in 200 and $400 \mu \mathrm{M}$ Cu-treated C. grandis leaves, and of G42 (LFNR2) was increased in $200 \mu \mathrm{M} \mathrm{Cu}$-treated C. grandis leaves. LFNR oxidizes ferredoxin $(\mathrm{Fd})$ to yield NADPH, which is utilized in various reactions such as lipid and $\mathrm{Chl}$ biosynthesis, $\mathrm{CO}_{2}$ fixation and stromal redox regulation. Arabidopsis fnr2 RNAi mutants had decreased concentrations of photosynthetic thylakoid proteins and Chls, and rate of carbon fixation relative to the wild type plants [37]. The abundances of Rubisco subunit binding-protein $\alpha$ subunit [chaperonin 60 subunit $\alpha 1$ (Cpn60 $\alpha 1)$; S11] involved in protein folding and Rubisco activase $1(\mathrm{G} 4, \mathrm{~S} 4$, and S14) involved in the activation of Rubisco were decreased in $400 \mu \mathrm{M}$ Cu-treated C. sinensis and/or C. grandis leaves. The abundance of Rubisco activase 1 (S14) was also decreased in 200 and $300 \mu \mathrm{M}$ Cu-treated C. sinensis leaves. Cpn60 1 is necessary for the folding of Rubisco large subunit (rbcL) and proper chloroplast development [38]. Rubisco activase-deficient transgenic tobacco plants had decreased Rubisco carbamylation and $\mathrm{CO}_{2}$ assimilation [39]. Also, the abundances of sedoheptulose-1,7-bisphosphatas (SBPase; S33) and phosphoribulokinase (PRK; S21) involved in Calvin cycle were decreased in $400 \mu \mathrm{M}$ Cu-treated C. granids leaves. The lower abundances of LFNR2 (G10), Cpn60 $\alpha 1$ (S11), Rubisco activase 1 (G4, S4, S9 and S14), PRK (S21) and SBPase (S33) agreed with our finding and previous report that excess $\mathrm{Cu}$-treated Citrus leaves had reduced $\mathrm{CO}_{2}$ assimilation and $\mathrm{Chl}$ concentrations (Figure 1) [5]. However, the abundances of LFNR2 (G42), SBPase (G6) and glyceraldehyde-3-phosphate dehydrogenase B (GAPB, G38) were increased in $200 \mu \mathrm{M}$ Cu-treated $C$. grandis leaves. This was agreement with the finding that $\mathrm{CO}_{2}$ assimilation 
displayed an upward trend in $200 \mu \mathrm{M}$ Cu-treated C. grandis leaves relative to controls (Figure 1). Similarly, the abundance of fructose-1,6-bisphosphatase, cytosolic (cyFBPase; G36), a major site for controlling sucrose synthesis, was increased in $200 \mu \mathrm{M} \mathrm{Cu}$-treated C. grandis. Strand et al. reported that photosynthesis was inhibited in antisense cyFBPase Arabidopsis mutants [40]. Also, the abundance of Cpn60 1 (G8) in C. grandis leaves was decreased and increased at 200 and $300 \mu \mathrm{M} \mathrm{Cu}$, respectively, and the abundance of Rubisco activase 1 (S10) was increased in $300 \mu \mathrm{M} \mathrm{Cu}$-treated C. sinensis leaves.

Carbonic anhydrase (CA, a $\mathrm{Zn}$-metalloenzyme) is required for $\mathrm{CO}_{2}$ assimilation in cotyledons. The abundance of CA (S3) was increased or unaffected by $\mathrm{Cu}$ supply in C. sinensis leaves. However, $\mathrm{CA}$ activity was reduced in Cu excess Brassica juncea [41]. The difference between CA abundance and activity could be explained by the $\mathrm{Cu}$-induced decrease in $\mathrm{Zn}$ level in C. sinensis [5], because its activity is regulated by $\mathrm{Zn}$ availability.

Mitochondrial $\mathrm{MDH}(\mathrm{mMDH})$ is necessary for $\mathrm{CO}_{2}$ and energy partitioning in leaves. Antisense $m M D H$ tomato plants displayed increased photosynthetic electron transport rate, $\mathrm{CO}_{2}$ assimilation, $\mathrm{g}_{\mathrm{s}}$ and growth rate, but decreased respiration rate [42]. The increased abundances of $\mathrm{mMDH}$ (S44 and S45) in $400 \mu \mathrm{M}$ Cu-treated C. sinensis leaves agreed with the report that $400 \mu \mathrm{M} \mathrm{Cu}$-treated C. sinensis seedlings had decreased growth, leaf $\mathrm{CO}_{2}$ assimilation and $\mathrm{g}_{\mathrm{s}}$, and impaired photosynthetic electron transport chain (PETC) [5]. Chloroplastic NADP-MDH, which catalyzes the excess NADPH produced through PETC and oxaloacetate to malate and $\mathrm{NADP}^{+}$, plays a key role in counteracting PETC over-reduction and in $\mathrm{H}_{2} \mathrm{O}_{2}$ signaling by exporting chloroplast $\mathrm{NADPH}$ to other cell compartments. Arabidopsis nadp-mdh mutants lacked the reversible inactivation of catalase activity and the concomitant accumulation of $\mathrm{H}_{2} \mathrm{O}_{2}$, but had a higher reduction state of the plastoquinone (PQ) pool when exposed to high light [43]. The decreased abundance of NADP-MDH (G17) in $400 \mu \mathrm{M} \mathrm{Cu-treated} \mathrm{C.} \mathrm{grandis} \mathrm{leaves}$ might contribute to the $\mathrm{Cu}$-induced inhibition of photosynthesis and the increased reduction of the PSII acceptor side, as indicated by the positive $\Delta \mathrm{J}$ - and $\Delta \mathrm{I}$-bands in $400 \mu \mathrm{M} \mathrm{Cu}$-treated C. grandis leaves [5]. However, $\mathrm{Cu}$ treatments increased or did not alter the abundance of cytosolic MDH (cyMDH; G37 and G39) in C. grandis leaves. cyMDH plays a key role in the transport of chloroplast or mitochondria NADPH to other cell compartments. Transgenic apple plants overexpressing an apple cyMDH gene displayed a higher stress-tolerance accompanied by increased reducing power, as indicated by increased concentrations of ASC and reduced glutathione (GSH) and ratios of ASC/dehydroascorbate (DHA), GSH/GSSG and NAD(P)H/NAD $(\mathrm{P})^{+}[44]$. Thus, the Cu-toxicity-induced increases of cyMDH abundances in $C$. grandis leaves might be an adaptive strategy.

Pentose phosphate pathway (PPP) provides NADPH for biosynthesis of GSH and maintenance of cellular redox state necessary to deal with oxidative stress. Arabidopsis PGL3 T-DNA insertion mutants with decreased flux through the plastidial PPP displayed a decrease in plant size and a lower cellular redox potential [45]. The increased abundance of probable 6-phosphogluconolactonase 4 (PGL4, an enzyme involved in PPP; G4) in 200 and $300 \mu \mathrm{M}$ Cu-treated C. grandis leaves agreed with the increased needs for ROS scavenging [5].

Triosephosphate isomerase (TPI), which catalyzing the reversible interconversion of glyceraldehydes 3-phosphate (GAP) and dihydroxyacetone phosphate (DHAP), may prevent the spontaneous degradation of DHAP into methylglyoxal (MG, a cytotoxic metabolite). TPI-deficiency led to increased generation of MG in red blood cells [46]. The decreased abundances of TPI (G29) in $400 \mu \mathrm{M}$ Cu-treated C. grandis leaves implied that MG formation was increased in these leaves, thus increasing ROS generation and lipid peroxidation [5].

The increased abundances of glucose-1-phosphate adenylyltransferase (APS, G24, and G25) in 200 and $300 \mu \mathrm{M} \mathrm{Cu}$-treated C. grandis leaves implied that starch biosynthesis was enhanced in these leaves. However, this way could not explain starch accumulation in $400 \mu \mathrm{M} \mathrm{Cu}$-treated C. grandis leaves, because APS abundance was not increased in these leaves. A weaker sink for the photosynthetic requirement due to $\mathrm{Cu}$ toxicity-induced inhibition of growth has been suggested to be responsible for the accumulation of nonstructural carbohydrates including starch in Cu-toxic Citrus leaves [5]. 
There is a close relation between energy availability and stress-tolerance [47]. An extra energy supply is necessary for stressed plants to fortify their tolerance. The increased abundances of ATP synthase subunit $\beta$ (G12) and ATP synthase $\gamma$ chain (G23) $300 \mu \mathrm{M} \mathrm{Cu}$-treated C. grandis leaves and bis(5'-adenosyl)-triphosphatase (Ap3A, G26) in $400 \mu \mathrm{M}$ Cu-treated C. grandis leaves suggested that ATP biosynthesis was enhanced in these leaves to meet the increased energy needs. Similar result has been obtained in Cu-stressed Elsholtzia splendens leaves [25].

To conclude, $\mathrm{Cu}$-toxicity might affect the abundances of proteins involved in PETC and $\mathrm{CO}_{2}$ assimilation, thus decreasing electron transport rate and $\mathrm{CO}_{2}$ assimilation. $\mathrm{Cu}$-toxic effects on PETC were more pronounced in $C$. sinensis leaves than those in $C$. grandis leaves.

\subsection{DAPs Related to Antioxidation and Detoxification}

Five (five) DAP spots involved in antioxidation and detoxification were identified in Cu-treated C. sinensis (C. grandis) leaves (Table 2). The striking Cu-mediated alteration was the big increase in GST (G40) abundance in Cu-treated C. grandis leaves. Dianthus superbus plants overexpressing GST were observed to biosynthesize phytochelatins (PCs), thus sequestering and detoxifying excess $\mathrm{Cu}$ [48]. Lambda class of GSTs could be used to enhance plant tolerance against various stresses including heavy metals [49]. However, the abundance of GST DHAR1 (G34), an enzyme having glutathione-dependent thiol transferase and DHA reductase (DHAR) activities, was decreased in 400 $\mu \mathrm{M}$ Cu-treated C. grandis leaves. SOD can rapidly dismutate $\mathrm{O}_{2}{ }^{-}$to $\mathrm{H}_{2} \mathrm{O}_{2}$ and protect organisms against oxidative damage. The increased abundances of $\mathrm{Cu} / \mathrm{Zn} \mathrm{SOD}$ (G21) and manganese (Mn) SOD (G33) in $400 \mu \mathrm{M}$ Cu-treated C. grandis leaves agreed with the report that $\mathrm{Cu}$ stress increased $\mathrm{Cu} / \mathrm{Zn}$ SOD and Mn SOD activity in Arabidopsis leaves [50]. Cu/Zn SOD abundance increased and Fe SOD abundance decreased in $\mathrm{Cu}$-sufficient Arabidopsis leaves, but the reverse was true in $\mathrm{Cu}$-limited leaves, which could save $\mathrm{Cu}$ for the biosynthesis of plastocyanin necessary for photosynthesis [51]. Thus, excess $\mathrm{Cu}$ increased the biosynthesis of $\mathrm{Cu} / \mathrm{Zn} \mathrm{SOD}$ by a direct effect of $\mathrm{Cu}$ on the gene for SOD, hence preventing a Cu-toxic effect on photosynthesis. Methyl viologen (mainly to enhance PSI-originated ROS formation) induced decrease of $\mathrm{F}_{\mathrm{v}} / \mathrm{F}_{\mathrm{m}}$ was more severe in aor [a chloroplastic NADPH-dependent alkenal/one oxidoreductase (AOR, At1g23740)] Arabidopsis mutants than in Col-0 plants, concluding that AOR played a role in the scavenging of stromal reactive carbonyls (RCs) generated under oxidative stress [52]. Therefore, the decreased abundance of quinone oxidoreductase-like protein At1g23740 (G16) in $400 \mu \mathrm{M}$ Cu-treated C. grandis might contribute to the Cu-induced inhibition of photosynthesis by lowering the photosynthetic electron transport rate.

The abundances of all the five DAP spots were decreased in Cu-treated C. sinensis leaves. The decreased abundances of three $\mathrm{H}_{2} \mathrm{O}_{2}$ detoxifying enzymes in 300 (S1) and 400 (S24 and S34) $\mu \mathrm{M}$ Cu-treated $C$. sinensis leaves agreed with the report that $\mathrm{H}_{2} \mathrm{O}_{2}$ production was increased in these leaves [5]. Cysteine (Cys) synthase (CS) catalyzes the final step for Cys biosynthesis in plants. The overexpression of CS conferred tolerance to Cd and selenium (Se) by over-production of Cys, GSH and presumably PCs, but not to $\mathrm{Cu}$ in transgenic tobacco plants [53]. PCs have been proven not to be the major factor responsible for plant $\mathrm{Cu}$-tolerance [54]. Thus, the $\mathrm{Cu}$-induced decrease of CS abundance (S20 and S39) might not lower the tolerance of $C$. sinensis seedlings to Cu.

To conclude, the antioxidation and detoxification system as a whole could not effectively protect Citrus leaves from Cu-toxicity-induced oxidative stress, as indicated by the increased $\mathrm{H}_{2} \mathrm{O}_{2}$ production and electrolyte leakage [5].

\subsection{Chaperones and Folding Catalysts}

Luminal binding protein (BiP) functions in both protein folding and endoplasmic reticulum (ER) quality control mechanism. Heterologous expression of an ER BiP gene alleviated Cd-induced ER stress and programmed cell death in transgenic tobacco BY-2 cells [55]. Transgenic tobacco plants overexpressing an ER chaperone BiP gene had enhanced Cd-tolerance accompanied by decreased level of ROS and increased level of GSH [56]. Thus, the increased abundance of luminal-binding 
protein $5(\mathrm{BiP5}, \mathrm{S16})$ might contribute to $\mathrm{Cu}$-tolerance of $C$. sinensis. Protein disulfide isomerase (PDI), which catalyzes thiol-disulfide interchange, is the most abundant oxidative protein folding catalyst and a multifunctional protein chaperone. PDI could serve as a $\mathrm{Cu}$ chelator or $\mathrm{Cu}$ delivering protein to protect cells against Cu-toxicity [57]. The increased abundance of probable PDI A6 (G15) in $400 \mu \mathrm{M}$ $\mathrm{Cu}$-treated C. grandis leaves might play a role in preventing these leaves from Cu-toxicity by binding $\mathrm{Cu}$ and/or decreasing oxidative damage. Like Cpn60 1 (G8), the abundance of $20 \mathrm{kDa}$ chaperonin (Cpn20, a co-chaperonin of CPN60; G12) was increased in $300 \mu \mathrm{M}$ Cu-treated C. grandis leaves. Cpn20 played a role in oxidative stress protection and chloroplast development via positively regulating the activation of Fe SOD [58]. Interestingly, the abundance of chaperonin CPN60-1 (G19 and S26) involved in the correct folding of imported proteins was decreased and increased in 200 and $300 \mu \mathrm{M}$ $\mathrm{Cu}$-treated C. grandis leaves, respectively, but was decreased in $400 \mu \mathrm{M} \mathrm{Cu}$-treated C. sinensis leaves. Also, the abundance of heat shock cognate $70 \mathrm{kDa}$ protein 2 (HSP70-2, G11) involved in the folding of de novo translocation of precursor proteins into organelles, and degradation of damaged protein under disadvantaged conditions was decreased in 300 and $400 \mu \mathrm{M} \mathrm{Cu}$-treated C. grandis leaves. These results demonstrate the involvement of chaperones and folding catalysts in the $\mathrm{Cu}$ tolerance and $\mathrm{Cu}$ toxicity of Citrus.

\subsection{DAPs Related to Signal Transduction}

Plant plasma membrane (PM) $\mathrm{H}^{+}$-ATPase activity can be regulated by 14-3-3 proteins involved in brassinosteroid (BR)-mediated signaling pathway [59]. The increased abundance of 14-3-3 protein 7 (G5) in $400 \mu \mathrm{M} \mathrm{Cu}$-treated C. grandis leaves agreed with the increased expression of a 14-3-3 gene in Fucus vesiculosus in response to moderately elevated level of $\mathrm{Cu}$ [60], and the increased activity of PM $\mathrm{H}^{+}$-ATPase in Cu-treated cucumber roots [61]. However, the abundance of 14-3-3 protein 6 (S7) was reduced in $200 \mu \mathrm{M}$ Cu-treated C. sinensis leaves.

Major pollen allergen, which is involved in abscisic acid (ABA)-activated signaling pathway, have high sequence homology to pathogenesis related (PR) proteins. The increased or unaltered abundance of major allergen Pru ar 1 (G27) in 200-400 $\mu$ M C. grandis leaves agreed with the elevated abundances of Bet v 1-Sc3 (PR-10c) and PvPR1 in Cu-stressed Betula pendula and bean leaves, respectively $[62,63]$. Annexins, a key element of $\mathrm{Ca}^{2+}$-signaling pathways, are involved in counteracting oxidative stress. Transgenic tobacco plants overexpressing an annexin displayed elevated total peroxidase activity, improved tolerance/resistance to Cd, oxidative stress and diseases, and increased message levels for several PR proteins [64]. The increased or unchanged abundance of annexin D1 (G22) in Cu-treated C. grandis leaves agreed with the increased abundance of annexin D1 in Cu-stressed Allium cepa roots [20]. Thus, Cu supply might enhance the resistance of $C$. grandis to diseases [65]. However, the abundance of major allergen Pru ar 1 (S35) was decreased in $200 \mu \mathrm{M}$ Cu-treated C. sinensis leaves.

Calreticulin (CRT), a crucial $\mathrm{Ca}^{2+}$-binding protein mainly in the ER, functions in $\mathrm{Ca}^{2+}$ signaling in response to stress in plants. The decreased abundance of CRT-1 (G3) agreed the decreased abundance of CRT in excess Cu-treated Ectocarpus siliculosus [66] and the decreased expression level of CRT in Mg-deficient Citrus reticulata leaves [67], because Mg concentration was decreased in Cu-stressed C. grandis leaves [5].

To conclude, hormone (ABA and $\mathrm{BR}$ )- and $\mathrm{Ca}^{2+}$-mediated signaling pathways might function in Citrus $\mathrm{Cu}$-tolerance and $\mathrm{Cu}$-toxicity. This was also supported by data suggesting that 28-homobrassinolide [41] and $\mathrm{Ca}$ [68] could alleviate plant $\mathrm{Cu}$-toxicity, and that a reciprocal cross-talk existed between $\mathrm{Cu}$ status and ABA metabolism and signaling in Arabidopsis [69].

\subsection{DAPs Related to Cellular Transport, Nucleic Acid and Cell Wall Metabolisms, and Cytoskeleton}

Ferritin can protect plant cells from Fe-toxicity by storing excess Fe in a non-toxic form in plant cells [70]. A characteristic of Cu-toxicity in Citrus leaves is Fe chlorosis [5,71]. The decreased abundance of ferritin-3 (S5) in 200-400 $\mu \mathrm{M}$ Cu-treated C. sinensis leaves agreed with the report that ferritin 
accumulation in plant cells increased under high Fe concentrations [72]. The decreased abundance of ferritin-3 might contribute to Fe homeostasis by lowering the chelation of Fe to ferritin.

Both $\alpha$ - and $\beta$-tubulins are the primary constituents of microtubules (MTs), one of the cytoskeletal components. MTs have been proposed to function in plant $\mathrm{Cu}$-toxicity and $\mathrm{Cu}$-tolerance. Song et al. found that the abundances of three protein spots-namely tubulin $\alpha-1$ chain, putative tubulin $\alpha-1$ chain and tubulin $\alpha-2$ chain, were decreased in excess $\mathrm{Cu}$-treated rice roots, concluding that the decreased accumulation of $\alpha$-tubulin might impair MT polymerization and alignment, thus influencing MT functions [18]. However, the abundance of tubulin $\beta-6$ chain (G7) in C. grandis leaves increased or did not alter in response to $\mathrm{Cu}$ supply, implying that MTs might be not impaired in these leaves. This might be related to the preferential accumulation of most $\mathrm{Cu}$ in the roots under $\mathrm{Cu}$-stress [5].

DNA helicases, which are ATP-dependent DNA unwinding enzymes, are involved in DNA repair, replication and recombination. Ectopic expression of a Medicago sativa helicase 1 (a homolog of the pea DNA helicase 4) gene conferred Arabidopsis tolerance to drought, salt and oxidative stress [73]. The decreased abundance of RuvB-like helicase 1 (S42) in $400 \mu \mathrm{M}$ Cu-treated C. sinensis leaves implied that DNA repair was impaired in these leaves.

The decreased or unaltered abundance of endochitinase 1 (G2) related to cell wall polysaccharide (macromolecule) catabolic process in Cu-treated C. grandis leaves implied that the level of cell wall polysaccharides might be increased in these leaves because of decreased degradation. This agreed with the increased concentration of total polysaccharide in the cell walls of Cu-treated Elsholtzia splendens roots [26]. However, the abundance of chitinase was enhanced in rice leaves treated with $100 \mu \mathrm{M} \mathrm{Cu}$ for $72 \mathrm{~h}$ [74]. Chitinase activity was not altered in pepper roots, stems, and cotyledons after 28 days of treatment with $50 \mu \mathrm{M} \mathrm{Cu}$ [65]. Thus, it seems that the effects of $\mathrm{Cu}$ on chitinase vary with plant species, $\mathrm{Cu}$ concentration, and time of exposure to $\mathrm{Cu}$.

\subsection{Other DAPs}

AdoHcy hydrolase, which catalyzes the reversible hydrolysis of AdoHcy to L-homocysteine and adenosine, plays a crucial role in maintaining methyl cycling via the removal of AdoHcy. Taddei et al. observed that AdoHcy hydrolase was induced by $\mathrm{Cu}$ stress in in vitro-cultured pith explants of Nicotiana glauca, suggesting that AdoHcy hydrolase played a crucial role in regulating Cu level and intracellular distribution [75]. B-induced alleviation of C. grandis Al-toxicity was accompanied by increased root expression of adenosylhomocysteinase-like [76]. The increased abundance of AdoHcy hydrolase (G32) in $200 \mu \mathrm{M}$ Cu-treated C. grandis leaves might contribute to their Cu-tolerance. However, its (S37) abundance was decreased in $400 \mu \mathrm{M}$ Cu-treated C. sinensis leaves.

Flavonoids can act as ROS scavengers, and inhibit ROS production by chelating metals. The decreased abundance of dihydroflavonol-4-reductase (DFR; S38) in $400 \mu \mathrm{M}$ Cu-treated C. sinensis leaves suggested that anthocyanin biosynthesis might be decreased in these leaves. This disagreed with the increased expression level of DFR in Cu-stressed rice leaves [77].

\section{Materials and Methods}

\subsection{Plant Materials}

Seedling culture and $\mathrm{Cu}$ treatments were made according to $\mathrm{Li}$ et al. [5]. Briefly, 6-week-old uniform seedlings of 'Xuegan' (Citrus sinensis) and 'Shatian pummelo' (Citrus grandis) were transported to $6 \mathrm{~L}$ pots (two plants per pot) filled with sand thoroughly washed with tap water, then grown in a greenhouse under natural conditions at Fujian Agriculture and Forestry University. Six weeks after transporting, seedlings were watered daily with freshly papered nutrient solution at a $\mathrm{Cu}$ concentration of 0.5 (Cu0.5, control), 200 (Cu200), 300 (Cu300), or 400 (Cu400) $\mu \mathrm{M}$ from $\mathrm{CuCl}_{2}$ until nutrients begin to flow out of the bottom hole of the pot ( $\sim 500 \mathrm{~mL}$ per pot). Nutrient solution $\mathrm{pH}$ was adjusted to 4.8 with $1 \mathrm{M} \mathrm{HCl}$ before supply. Six months after $\mathrm{Cu}$ treatments, the fully expanded (about 7-week-old) leaves were used for all measurements. Firstly, leaf gas exchange was measured. Then, leaves (winged 
leaves, petioles and midribs removed) were taken at a sunny noon and immediately frozen in liquid $\mathrm{N}_{2}$. All samples were stored at $-80^{\circ} \mathrm{C}$ until extraction of proteins and total RNA. These seedlings unused for the collection of leaves were used for the measurements of plant dry weight (DW) and leaf Cu.

\subsection{Measurements of Plant DW, and Leaf Gas Exchange and Cu Concentration}

Root, stem, and leaf DW were weighted after being washed with tap water and dried to a constant weight at $70{ }^{\circ} \mathrm{C}(\sim 48 \mathrm{~h})$ [78].

Gas exchange was measured with a CIARS-2 portable photosynthesis system (PP systems, Herts, UK) at a controlled light intensity of $\sim 1000 \mu \mathrm{mol} \mathrm{m} \mathrm{m}^{-2} \mathrm{~s}^{-1}$ and a controlled $\mathrm{CO}_{2}$ concentration of $\sim 380 \mu \mathrm{mol} \mathrm{mol} \mathrm{m}^{-1}$ between 9:30 and 12:30 a.m. on a sunny day [79].

Leaf $\mathrm{Cu}$ was determined with a NexION 300X Inductively Coupled Plasma Mass Spectrometer (ICP-MS, PerkinElmer, Shelton, CT, USA).

\subsection{Leaf Protein Extraction, 2-DE and Image Analysis}

About $1 \mathrm{~g}$ of frozen leaves harvested equally from four seedlings (one seedling per pot) was mixed as one biological replicate. There were three biological replicates per treatment (a total of 12 seedlings from 12 pots). Proteins were extracted using a phenol extraction procedure [80] and their concentration was measured as described by Bradford [81]. 2-DE was performed according to Sang et al. [82]. Stained gels were scanned with an Epson Scanner (Seiko Epson Corporation, Japan) at a resolution of 300 dpi. Images were analyzed with PDQuest version 8.0.1 (BioRad, Hercules, CA, USA), including background subtraction, normalization, spot detection, matching, Gaussian fitting and gel alignment [83]. A fold change of $>1.5$ or $<0.67$ was set to determine DAP spots in addition to a $p$-value $<0.05$. After being visually checked and manually excised from gels, all DAP spots were submitted to MALDI-TOF/TOF-MS-based identification.

\subsection{MALDI-TOF/TOF-MS-Based Protein Identification and Bioinformatic Analysis}

Peptide identification was carried out on an AB SCIEX 5800 TOF/TOF plus MS (AB SCIEX, Shanghai, China) as described by Peng et al. [83]. After being processed with TOF/TOF Explorer ${ }^{\mathrm{TM}}$ Software (AB SCIEX, Shanghai, China) in a default mode, all acquired spectra were submitted to MASCOT (Version 2.3, Matrix Science Inc., Boston, MA) by GPS Explorer (Version 3.6) for the search of C. sinensis databases (http://citrus.hzau.edu.cn/orange/index.php) using following search parameters: trypsin cleavage with one missed, MS tolerance of 100 ppm and MS/MS tolerance of 0.6 Da. At least two of matched peptides were necessary for each protein. Protein identifications were accepted if MASCOT score was $\geq 70$, and the sequence coverage was $\geq 20 \%$ or the number of matched peptides (NMP) was $\geq$ five [84,85]. DAPs were classified according to KEGG (http://www.kegg.jp/), GO (http://www.geneontology.org/) and Uniprot (http://www.uniprot.org/) databases [86,87].

\subsection{KEGG Pathway Analysis of DAPs}

KEGG pathway was analyzed using KOBAS 3.0 (Peking University, Beijing, China). Pathways were considered as significantly enriched if the corrected $p$-value was less than 0.05

\section{6. $q R T-P C R$ Analysis}

Total RNA were extracted from $\sim 300 \mathrm{mg}$ frozen of leaves (mixed sample from four seedlings, one seedling per pot) using Recalcirtant Plant Total RNA Extraction Kit (Bioteke Corporation, Beijing, China). There were three biological replicates per treatment (a total of 12 seedlings from 12 pots). The sequences of specific primers designed using Primer Primier Version 5.0 (PREMIER Biosoft International, CA, USA), were listed in Table S4. qRT-PCR was performed with three biological and two technical replicates [86]. Two Citrus genes: U4/U6 small nuclear ribonucleoprotein PRP31 (PRP31, 
Cs7g08440.1) and actin (Cs1g05000.1) were used as internal standards and $0.5 \mu \mathrm{M}$ Cu-treated leaves were used as reference (set as 1 ).

\subsection{Data Analysis}

There were 15 pots (30 seedlings) per treatment in a completely randomized design. Results were presented as the mean \pm SE for $n=3-10$. Eight means [two (species) $\times$ four (Cu levels)] were tested by two ANOVA followed by the least significant difference at $p<0.05$ level.

Pearson correlation analysis and principal component analysis (PCA) for all identified DAP spots were made using SPSS (version 17.0, IBM, NY, USA) [88].

\subsection{Data Deposit}

The mass spectrometry proteomics data have been deposited to the ProteomeXchange Consortium via the PRIDE partner repository with the dataset identifier PXD017049.

\section{Conclusions}

In this study, a 2-DE based MS approach was used to investigate Cu-toxicity-responsive proteins in Citrus leaves. Forty-one and 37 DAP spots were identified in 200, 300 and/or $400 \mu \mathrm{M} \mathrm{Cu}$-treated C. grandis and C. sinensis leaves, respectively. Over $50 \%$ of these DAPs were involved in photosynthesis, carbohydrate, and energy metabolism, followed by antioxidation and detoxification, protein folding and assembly (viz., chaperones and folding catalysts), and signal transduction. More than $80 \%$ of these DAPs were identified only in C. grandis or C. sinensis leaves. More (Less) DAPs increased in abundances than DAPs decreased in abundances were identified in Cu-treated C. grandis (C. sinensis) leaves. Impaired PETC and decreased abundances of proteins involved in $\mathrm{CO}_{2}$ assimilation might be responsible for the $\mathrm{Cu}$-induced inhibition of photosynthesis. Cu-toxicity affected the PETC more in C. sinensis leaves than in C. grandis leaves. DAPs related to antioxidation and detoxification, protein folding and assembly (viz., chaperones and folding catalysts), and signal transduction might be involved in Citrus Cu-toxicity and Cu-tolerance. Also, we identified some new DAPs (viz., LFNR2, SBPase, probable PGL4, ferritin, AdoHcy hydrolase and abscisic stress-ripening protein 1-like) that were not reported in leaves and/or roots (Figure 5). In conclusion, this study revealed some novel mechanisms on $\mathrm{Cu}$-toxicity and $\mathrm{Cu}$-tolerance in plants. 


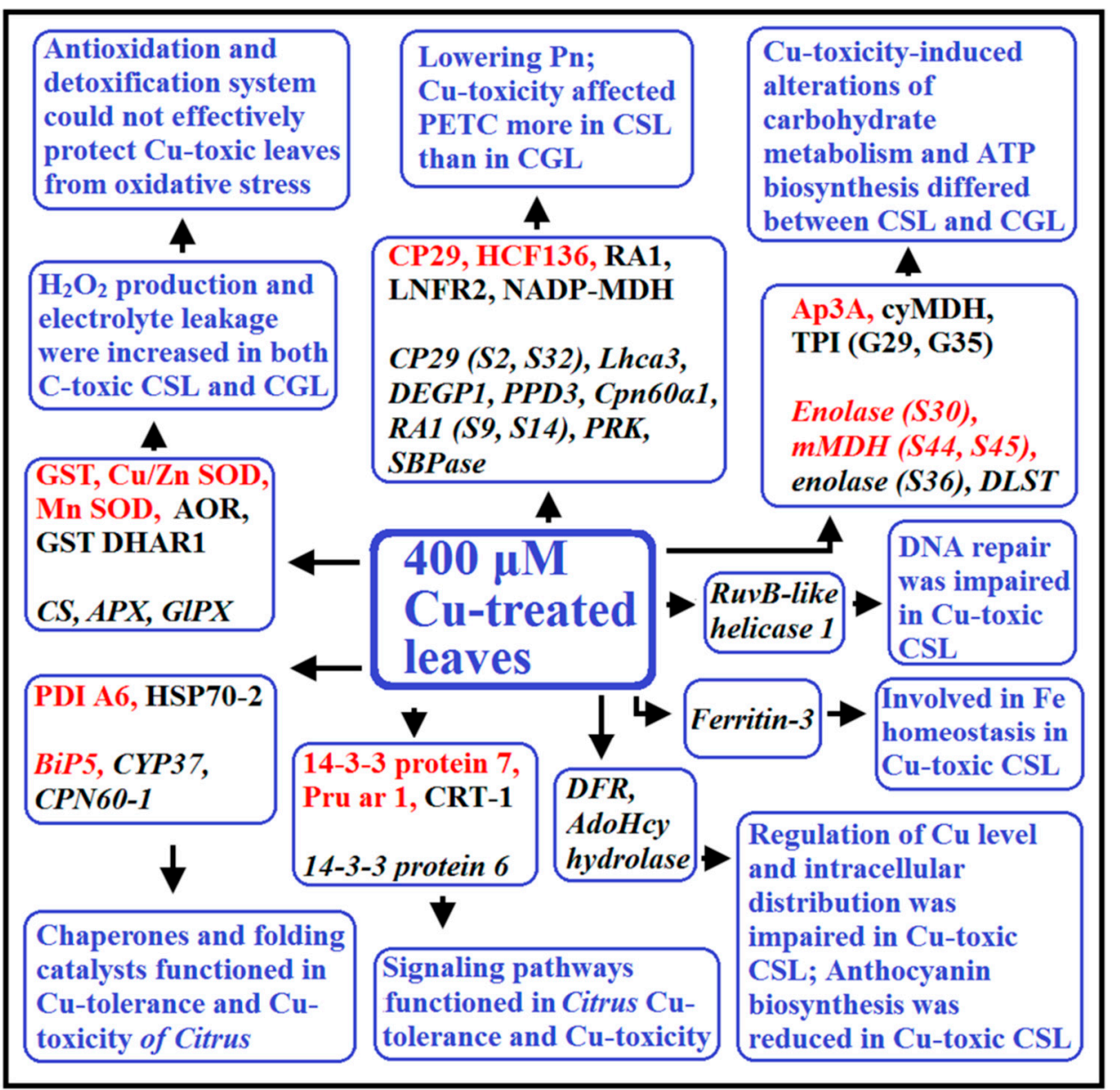

Figure 5. A possible model displaying the differentially abundant proteins (DAPs) in $400 \mu \mathrm{MCu}$-treated Citrus grandis leaves (CGL) and Citrus sinensis leaves (CSL). In this Figure, plain format and italics were used for Cu-toxic CGL and CSL, respectively. Red: DAPs increased in abundance; Black: DAPs decreased in abundances; APX, L-ascorbate peroxidase; DLST: dihydrolipoyllysine-residue succinyltransferase component of 2-oxoglutarate dehydrogenase complex 2; GIPX: glutathione peroxidase; Pn: photosynthesis; RA1: Rubisco activase A1.

Supplementary Materials: The following are available online at http://www.mdpi.com/2223-7747/9/3/291/s1, Figure S1: Excess $\mathrm{Cu}$ effects on growth of Citrus grandis (A) and Citrus sinensis (B), Figure S2: Excess Cu effects on root (A), stem (B), leaf (C) and whole plant (D) dry weight (DW) in Citrus grandis and Citrus sinensis seedlings, Figure S3: 2-DE images of proteins extracted from 0.5 (A, E, I, M), 200 (B, F, J, N) 300 (C, G, K, O) and 400 (D, H, L, P) $\mathrm{Cu}$-treated Citrus grandis (A-D and I-L) and Citrus sinensis (E-H and M-P) leaves for the other two replicates, Figure S4: Close-up views of 24 DAP spots in 200, 300 and $400 \mu \mathrm{M} \mathrm{Cu}$-treated Citrus sinensis and Citrus grandis leaves, Figure S5: Venn diagram analysis of Cu-responsive proteins in Citrus grandis and Citrus sinensis leaves, Figure S6: Significantly enriched (A-E and H) and the most enriched (F-G) KEGG pathways for annotated differentially abundant proteins (DAPs) in Cu-treated Citrus grandis (A-D) and Citrus sinensis (E-H) leaves, Figure S7: Matrices of Pearson correlation coefficients for differentially abundant proteins (DAPs) in Citrus grandis (A) and Citrus sinensis (B) leaves, Figure S8: Relative expression levels of genes encoding 22 differentially abundant proteins (DAPs) identified in $400 \mu \mathrm{M}$ Cu-treated Citrus grandis (G3, G9, G10, G11, G14, G26, G29, G33, G34 and G35) and Citrus sinensis (S2, S5, S9, S16, S17, S23, S24, S30, S32, S33, S37 and S43) leaves using PRPF31 (A) and actin (B) as internal standards, Table S1: Master list of proteins identified in MALDI TOF/TOF MS from 200, 300 and or 400 $\mu \mathrm{M}$ Cu-treated Citrus sinensis and Citrus grandis leaves using 2DE and DIGE experiments, Table S2: Principal component analysis (PCA) for copper-responsive proteins in Citrus sinensis leaves, Table S3: Principal component analysis (PCA) for copper-responsive proteins in Citrus grandis leaves, Table S4: Specific primer pairs used for qRT-PCR analysis.

Author Contributions: Conceptualization, W.-L.H. and L.-S.C.; methodology, W.-L.H., L.-T.Y. and L.-S.C.; software, W.-L.H. and Z.-R.H.; validation, W.-L.H.; formal analysis, W.-L.H. and C.-L.D.; investigation, W.-L.H., F.-L.W., H.-Y.H. and W.-T.H.; resources, W.-L.H. and L.-S.C.; data curation, W.-L.H. and L.-T.Y.; writing-original draft 
preparation, W.-L.H.; writing-review and editing, L.-S.C.; supervision, L.-T.Y. and L.-S.C.; project administration, L.-S.C.; funding acquisition, L.-S.C. All authors have read and agreed to the published version of the manuscript.

Funding: This research was funded by the National Key Research and Development Program of China (2018YFD1000305), and the earmarked fund for China Agriculture Research System (CARS27). The APC was funded by the National Key Research and Development Program of China (2018YFD1000305).

Conflicts of Interest: The authors declare no conflict of interest. The funders had no role in the design of the study; in the collection, analyses, or interpretation of data; in the writing of the manuscript, or in the decision to publish the results.

\section{References}

1. Adrees, M.; Ali, S.; Rizwan, M.; Ibrahim, M.; Abbas, F.; Farid, M.; Zia-ur-Rehman, M.; Irshad, M.K.; Bharwana, S.A. The effect of excess copper on growth and physiology of important food crops: A review. Environ. Sci. Pollut. Res. 2015, 22, 8148-8162. [CrossRef]

2. Fan, J.; He, Z.; Ma, L.Q.; Stoffella, P.J. Accumulation and availability of copper in Citrus grove soils as affected by fungicide application. J. Soils Sediments 2011, 11, 639-648. [CrossRef]

3. Mozaffari, M.; Alva, A.K.; Chen, E.Q. Relation of copper extractable from soil and $\mathrm{pH}$ to copper content and growth of two Citrus rootstocks. Soil Sci. 1996, 161, 786-792. [CrossRef]

4. Yuan, M.; Li, Y.; Zhang, C.; Wang, J.; Li, S.; Fu, X.; Ling, L.; Cao, L.; Peng, L. Review of research on copper stress in Citrus. J. Fruit Sci. 2018, 35, 347-357.

5. Li, Q.; Chen, H.-H.; Qi, Y.P.; Ye, X.; Yang, L.T.; Huang, Z.R.; Chen, L.S. Excess copper effects on growth, uptake of water and nutrients, carbohydrates, and PSII photochemistry revealed by OJIP transients in Citrus seedlings. Environ. Sci. Pollut. Res. 2019, 26, 30188-30205. [CrossRef] [PubMed]

6. Burkhead, J.L.; Reynolds, K.A.G.; Abdel-Ghany, S.E.; Cohu, C.M.; Pilon, M. Copper homeostasis. New Phytol. 2009, 182, 799-816. [CrossRef]

7. Ambrosini, V.G.; Rosa, D.J.; Basso, A.; Borghezan, M.; Pescador, R.; Miotto, A.; George de Melo, W.B.; de Sousa Soares, C.R.F.; Comin, J.J.; Brunetto, G. Liming as an ameliorator of copper toxicity in black oat (Avena strigosa Schreb.). J. Plant Nutr. 2017, 40, 404-416. [CrossRef]

8. Hippler, F.W.R.; Boaretto, R.M.; Dovis, V.L.; Quaggio, J.A.; Azevedo, R.A.; Mattos, D., Jr. Oxidative stress induced by $\mathrm{Cu}$ nutritional disorders in Citrus depends on nitrogen and calcium availability. Sci. Rep. 2018, 8, 1641. [CrossRef]

9. Zhang, L.L.; He, X.J.; Chen, M.; An, R.D.; An, X.L.; Li, J. Responses of nitrogen metabolism to copper stress in Luffa cylindrica roots. J. Soil Sci. Plant Nutr. 2014, 14, 616-624. [CrossRef]

10. Fürtig, K.; Pavelic, D.; Brunold, C.; Brändle, R. Copper-and iron-induced injuries in roots and rhizomes of reed (Phragmites australis). Limnologica 1999, 29, 60-63. [CrossRef]

11. Lequeux, H.; Hermans, C.; Lutts, S.; Verbruggen, N. Response to copper excess in Arabidopsis thaliana: Impact on the root system architecture, hormone distribution, lignin accumulation and mineral profile. Plant Physiol. Biochem. 2010, 48, 673-682. [CrossRef]

12. Colzi, I.; Doumett, S.; Del Bubba, M.; Fornaini, J.; Arnetoli, M.; Gabbrielli, R.; Gonnelli, C. On the role of the cell wall in the phenomenon of copper tolerance in Silene paradoxa L. Environ. Exp. Bot. 2011, 72, 77-83. [CrossRef]

13. Kováčik, J.; Klejdus, B.; Hedbavny, J.; Štork, F.; Bačkor, M. Comparison of cadmium and copper effect on phenolic metabolism, mineral nutrients and stress-related parameters in Matricaria chamomilla plants. Plant Soil 2009, 320, 231-242. [CrossRef]

14. Yruela, I. Copper in plants: Acquisition, transport and interactions. Funct. Plant. Biol. 2009, 36, 409-430. [CrossRef]

15. Greenbaum, D.; Colangelo, C.; Williams, K.; Gerstein, M. Comparing protein abundance and mRNA expression levels on a genomic scale. Genome Biol. 2003, 4, 117-124. [CrossRef]

16. Rose, J.K.; Bashir, S.; Giovannoni, J.J.; Jahn, M.M.; Saravanan, R.S. Tackling the plant proteome: Practical approaches, hurdles and experimental tools. Plant J. 2004, 39, 715-733. [CrossRef] [PubMed]

17. Chen, C.; Song, Y.; Zhuang, K.; Li, L.; Xia, Y.; Shen, Z. Proteomic analysis of copper-binding proteins in excess copper-stressed roots of two rice (Oryza sativa L.) varieties with different Cu tolerances. PLoS ONE 2015, 10, e0125367. [CrossRef] 
18. Song, Y.; Cui, J.; Zhang, H.; Wang, G.; Zhao, F.J.; Shen, Z. Proteomic analysis of copper stress responses in the roots of two rice (Oryza sativa L.) varieties differing in Cu tolerance. Plant Soil 2013, 366, 647-658. [CrossRef]

19. Zhang, H.; Lian, C.; Shen, Z. Proteomic identification of small, copper-responsive proteins in germinating embryos of Oryza sativa. Ann. Bot. 2009, 103, 923-930. [CrossRef]

20. Qin, R.; Ning, C.; Björn, L.O.; Li, S. Proteomic analysis of Allium cepa var. agrogarum L. roots under copper stress. Plant Soil 2016, 401, 197-212.

21. Wang, C.; Wang, J.; Wang, X.; Xia, Y.; Chen, C.; Shen, Z.; Chen, Y. Proteomic analysis on roots of Oenothera glazioviana under copper-stress conditions. Sci. Rep. 2017, 7, 10589. [CrossRef] [PubMed]

22. Gill, T.; Dogra, V.; Kumar, S.; Ahuja, P.S.; Sreenivasulu, Y. Protein dynamics during seed germination under copper stress in Arabidopsis over-expressing Potentilla superoxide dismutase. J. Plant Res. 2012, 125, 165-172. [CrossRef]

23. Bona, E.; Marsano, F.; Cavaletto, M.; Berta, G. Proteomic characterization of copper stress response in Cannabis sativa roots. Proteomics 2007, 7, 1121-1130. [CrossRef] [PubMed]

24. Hego, E.; Bes, C.M.; Bedon, F.; Palagi, P.M.; Chaumeil, P.; Barré, A.; Claverol, S.; Dupuy, J.W.; Bonneu, M.; Lalanne, C.; et al. Differential accumulation of soluble proteins in roots of metallicolous and nonmetallicolous populations of Agrostis capillaris L. exposed to Cu. Proteomics 2014, 14, 1746-1758. [CrossRef]

25. Li, F.; Shi, J.; Shen, C.; Chen, G.; Hu, S.; Chen, Y. Proteomic characterization of copper stress response in Elsholtzia splendens roots and leaves. Plant Mol. Biol. 2009, 71, 251-263. [CrossRef] [PubMed]

26. Liu, T.; Shen, C.; Wang, Y.; Huang, C.; Shi, J. New insights into regulation of proteome and polysaccharide in cell wall of Elsholtzia splendens in response to copper stress. PLoS ONE 2014, 9, e109573. [CrossRef]

27. Roy, S.K.; Kwon, S.J.; Cho, S.W.; Kamal, A.H.M.; Kim, S.W.; Sarker, K.; Oh, M.W.; Lee, M.S.; Chung, K.Y.; Xin, Z.; et al. Leaf proteome characterization in the context of physiological and morphological changes in response to copper stress in sorghum. Biometals 2016, 29, 495-513. [CrossRef]

28. Roy, S.K.; Cho, S.W.; Kwon, S.J.; Kamal, A.H.M.; Lee, D.G.; Sarker, K.; Moon-Soon Lee, M.S.; Xin, Z.; Woo, S.H. Proteome characterization of copper stress responses in the roots of sorghum. Biometals 2017, 30, 765-785. [CrossRef]

29. Li, G.; Peng, X.; Xuan, H.; Wei, L.; Yang, Y.; Guo, T.; Kang, G. Proteomic analysis of leaves and roots of common wheat (Triticum aestivum L.) under copper-stress conditions. J. Proteome Res. 2013, 12, 4846-4861. [CrossRef]

30. Alotaibi, M.O.; Mohammed, A.E.; Almutairi, T.A.; Elobeid, M.M. Morpho-physiological and proteomic analyses of Eucalyptus camaldulensis as a bioremediator in copper-polluted soil in Saudi Arabia. Plants 2019, 8, 43. [CrossRef]

31. Damkjær, J.T.; Kereiche, S.; Johnson, M.P.; Kovacs, L.; Kiss, A.Z.; Boekema, E.J.; Ruban, A.V.; Horton, P.; Jansson, S. The photosystem II light-harvesting protein Lhcb3 affects the macrostructure of photosystem II and the rate of state transitions in Arabidopsis. Plant Cell 2009, 21, 3245-3256. [CrossRef] [PubMed]

32. Kapri-Pardes, E.; Naveh, L.; Adam, Z. The thylakoid lumen protease Deg1 is involved in the repair of photosystem II from photoinhibition in Arabidopsis. Plant Cell 2007, 19, 1039-1047. [CrossRef] [PubMed]

33. Bergantino, E.; Dainese, P.; Cerovic, Z.; Sechi, S.; Bassi, R. A post-translational modification of the PSII subunit CP29 protects maize from cold stress. J. Biol. Chem. 1995, 270, 8474-8481. [CrossRef] [PubMed]

34. Betterle, N.; Ballottari, M.; Baginsky, S.; Bassi, R. High light-dependent phosphorylation of photosystem II inner antenna CP29 in monocots is STN7 independent and enhances nonphotochemical quenching. Plant Physiol. 2015, 167, 457-471. [CrossRef]

35. Farinati, S.; DalCorso, G.; Bona, E.; Corbella, M.; Lampis, S.; Cecconi, D.; Polati, R.; Berta, G.; Vallini, G.; Furini, A. Proteomic analysis of Arabidopsis halleri shoots in response to the heavy metals cadmium and zinc and rhizosphere microorganisms. Proteomics 2009, 9, 4837-4850. [CrossRef]

36. Lundin, B.; Hansson, M.; Schoefs, B.; Vener, A.V.; Spetea, C. The Arabidopsis PsbO2 protein regulates dephosphorylation and turnover of the photosystem II reaction centre D1 protein. Plant J. 2007, 49, 528-539. [CrossRef]

37. Lintala, M.; Allahverdiyeva, Y.; Kangasjärvi, S.; Lehtimäki, N.; Keränen, M.; Rintamäki, E.; Aro, E.M.; Mulo, P. Comparative analysis of leaf-type ferredoxin-NADP ${ }^{+}$oxidoreductase isoforms in Arabidopsis thaliana. Plant J. 2009, 57, 1103-1115. [CrossRef]

38. Kim, S.R.; Yang, J.I.; An, G. OsCpn60 $\alpha 1$, encoding the plastid chaperonin $60 \alpha$ subunit, is essential for folding of rbcL. Mol. Cells 2013, 35, 402-409. [CrossRef] [PubMed] 
39. Mate, C.J.; von Caemmerer, S.; Evans, J.R.; Hudson, G.S.; Andrews, T.J. The relationship between $\mathrm{CO}_{2}$-assimilation rate, Rubisco carbamylation and Rubisco activase content in activase-deficient transgenic tobacco suggests a simple model of activase action. Planta 1996, 198, 604-613. [CrossRef]

40. Strand, Å.; Zrenner, R.; Trevanion, S.; Stitt, M.; Gustafsson, P.; Gardeström, P. Decreased expression of two key enzymes in the sucrose biosynthesis pathway, cytosolic fructose-1, 6-bisphosphatase and sucrose phosphate synthase, has remarkably different consequences for photosynthetic carbon metabolism in transgenic Arabidopsis thaliana. Plant J. 2000, 23, 759-770.

41. Fariduddin, Q.; Yusuf, M.; Hayat, S.; Ahmad, A. Effect of 28-homobrassinolide on antioxidant capacity and photosynthesis in Brassica juncea plants exposed to different levels of copper. Environ. Exp. Bot. 2009, 66, 418-424. [CrossRef]

42. Nunes-Nesi, A.; Carrari, F.; Lytovchenko, A.; Smith, A.M.; Loureiro, M.E.; Ratcliffe, R.G.; Sweetlove, L.J.; Fernie, A.R. Enhanced photosynthetic performance and growth as a consequence of decreasing mitochondrial malate dehydrogenase activity in transgenic tomato plants. Plant Physiol. 2005, 137, 611-622. [CrossRef] [PubMed]

43. Heyno, E.; Innocenti, G.; Lemaire, S.D.; Issakidis-Bourguet, E.; Krieger-Liszkay, A. Putative role of the malate valve enzyme NADP-malate dehydrogenase in $\mathrm{H}_{2} \mathrm{O}_{2}$ signalling in Arabidopsis. Philos. Trans. R. Soc. B 2014, 369, 20130228. [CrossRef] [PubMed]

44. Wang, Q.J.; Sun, H.; Dong, Q.L.; Sun, T.Y.; Jin, Z.X.; Hao, Y.J.; Yao, Y.X. The enhancement of tolerance to salt and cold stresses by modifying the redox state and salicylic acid content via the cytosolic malate dehydrogenase gene in transgenic apple plants. Plant Biotech. J. 2016, 14, 1986-1997. [CrossRef] [PubMed]

45. Xiong, Y.; DeFraia, C.; Williams, D.; Zhang, X.; Mou, Z. Characterization of Arabidopsis 6-phosphogluconolactonase T-DNA insertion mutants reveals an essential role for the oxidative section of the plastidic pentose phosphate pathway in plant growth and development. Plant Cell Physiol. 2009, 50, 1277-1291. [CrossRef] [PubMed]

46. Ahmed, N.; Battah, S.; Karachalias, N.; Babaei-Jadidi, R.; Horányi, M.; Baróti, K.; Hollan, S.; Thornalley, P.J. Increased formation of methylglyoxal and protein glycation, oxidation and nitrosation in triosephosphate isomerase deficiency. Biochim. Biophys. Acta-Mol. Basis Dis. 2003, 1639, 121-132. [CrossRef]

47. Baena-González, E.; Sheen, J. Convergent energy and stress signaling. Trends Plant Sci. 2008, 13, 474-482. [CrossRef]

48. Lim, J.D.; Hahn, S.J.; Yu, C.Y.; Chung, I.M. Expression of the glutathione S-transferase gene (NT107) in transgenic Dianthus superbus. Plant Cell Tiss. Org. Cult. 2005, 80, 277-286. [CrossRef]

49. Kumar, S.; Asif, M.H.; Chakrabarty, D.; Tripathi, R.D.; Dubey, R.S.; Trivedi, P.K. Expression of a rice Lambda class of glutathione S-transferase, OsGSTL2, in Arabidopsis provides tolerance to heavy metal and other abiotic stresses. J. Hazard. Mater. 2013, 248, 228-237. [CrossRef]

50. Drążkiewicz, M.; Skórzyńska-Polit, E.; Krupa, Z. The redox state and activity of superoxide dismutase classes in Arabidopsis thaliana under cadmium or copper stress. Chemosphere 2007, 67, 188-193. [CrossRef]

51. Cohu, C.M.; Pilon, M. Regulation of superoxide dismutase expression by copper availability. Physiol. Plant. 2007, 129, 747-755. [CrossRef]

52. Yamauchi, Y.; Hasegawa, A.; Mizutani, M.; Sugimoto, Y. Chloroplastic NADPH-dependent alkenal/one oxidoreductase contributes to the detoxification of reactive carbonyls produced under oxidative stress. FEBS Lett. 2012, 586, 1208-1213. [CrossRef] [PubMed]

53. Kawashima, C.G.; Noji, M.; Nakamura, M.; Ogra, Y.; Suzuki, K.T.; Saito, K. Heavy metal tolerance of transgenic tobacco plants over-expressing cysteine synthase. Biotechnol. Let. 2004, 26, 153-157. [CrossRef] [PubMed]

54. Lee, S.; Kang, B.S. Phytochelatin is not a primary factor in determining copper tolerance. J. Plant Biol. 2005, 48, 32-38. [CrossRef]

55. Xu, H.; Xu, W.; Xi, H.; Ma, W.; He, Z.; Ma, M. The ER luminal binding protein (BiP) alleviates $\mathrm{Cd}^{2+}$-induced programmed cell death through endoplasmic reticulum stress-cell death signaling pathway in tobacco cells. J. Plant Physiol. 2013, 170, 1434-1441. [CrossRef]

56. Guan, C.; Jin, C.; Ji, J.; Wang, G.; Li, X. LcBiP, a endoplasmic reticulum chaperone binding protein gene from Lycium chinense, confers cadmium tolerance in transgenic tobacco. Biotechnol. Prog. 2015, 31, 358-368. [CrossRef] 
57. Narindrasorasak, S.; Yao, P.; Sarkar, B. Protein disulfide isomerase, a multifunctional protein chaperone, shows copper-binding activity. Biochem. Biophys. Res. Commun. 2003, 311, 405-414. [CrossRef]

58. Kuo, W.Y.; Huang, C.H.; Liu, A.C.; Cheng, C.P.; Li, S.H.; Chang, W.C.; Weiss, C.; Azem, A.; Jinn, T.L. CHAPERONIN 20 mediates iron superoxide dismutase (Fe SOD) activity independent of its co-chaperonin role in Arabidopsis chloroplasts. New Phytol. 2013, 197, 99-110. [CrossRef]

59. Palmgren, M.G. Plant plasma membrane $\mathrm{H}^{+}$-ATPases: Powerhouses for nutrient uptake. Annu. Rev. Plant Physiol. Plant Mol. Biol. 2001, 52, 817-845. [CrossRef]

60. Owen, J.R.; Morris, C.A.; Nicolaus, B.; Harwood, J.L.; Kille, P. Induction of expression of a 14-3-3 gene in response to copper exposure in the marine alga, Fucus vesiculosus. Ecotoxicology 2012, 21, 124-138. [CrossRef] [PubMed]

61. Janicka-Russak, M.; Kabała, K.; Burzyński, M. Different effect of cadmium and copper on $\mathrm{H}^{+}$-ATPase activity in plasma membrane vesicles from Cucumis sativus roots. J. Exp. Bot. 2012, 63, 4133-4142. [CrossRef] [PubMed]

62. Cuypers, A.; Koistinen, K.M.; Kokko, H.; Kärenlampi, S.; Auriola, S.; Vangronsveld, J. Analysis of bean (Phaseolus vulgaris L.) proteins affected by copper stress. J. Plant Physiol. 2005, 162, 383-392. [CrossRef]

63. Utriainen, M.; Kokko, H.; Auriola, S.; Sarrazin, O.; Kärenlampi, S. PR-10 protein is induced by copper stress in roots and leaves of a $\mathrm{Cu} / \mathrm{Zn}$ tolerant clone of birch, Betula pendula. Plant Cell Environ. 1998, 21, 821-828. [CrossRef]

64. Jami, S.K.; Clark, G.B.; Turlapati, S.A.; Handley, C.; Roux, S.J.; Kirti, P.B. Ectopic expression of an annexin from Brassica juncea confers tolerance to abiotic and biotic stress treatments in transgenic tobacco. Plant Physiol. Biochem. 2008, 46, 1019-1030. [CrossRef] [PubMed]

65. Chmielowska, J.; Veloso, J.; Gutierrez, J.; Silvar, C.; Díaz, J. Cross-protection of pepper plants stressed by copper against a vascular pathogen is accompanied by the induction of a defence response. Plant Sci. 2010, 178, 176-182. [CrossRef]

66. Ritter, A.; Ubertini, M.; Romac, S.; Gaillard, F.; Delage, L.; Mann, A.; Cock, J.M.; Tonon, T.; Correa, J.A.; Potin, P. Copper stress proteomics highlights local adaptation of two strains of the model brown alga Ectocarpus siliculosus. Proteomics 2010, 10, 2074-2088. [CrossRef] [PubMed]

67. Jin, X.L.; Ma, C.L.; Yang, L.T.; Chen, L.S. Alterations of physiology and gene expression due to long-term magnesium-deficiency differ between leaves and roots of Citrus reticulata. J. Plant Physiol. 2016, 198, 103-115. [CrossRef] [PubMed]

68. Zhai, F.Q.; Wang, X.L.; Hua, J.M.; Si, J.Y.; Feng, K. Copper toxicity on seedlings of wheat and the detoxification of calcium. J. Agro-Environ. Sci. 2006, 26, 694-698.

69. Carrió-Seguí, À.; Romero, P.; Sanz, A.; Peñarrubia, L. Interaction between ABA signaling and copper homeostasis in Arabidopsis thaliana. Plant Cell Physiol. 2016, 57, 1568-1582.

70. Briat, J.F. Roles of ferritin in plants. J. Plant Nutr. 1996, 19, 1331-1342. [CrossRef]

71. Alva, A.K.; Chen, E.Q. Effects of external copper concentrations on uptake of trace elements by Citrus seedlings. Soil Sci. 1995, 159, 59-64. [CrossRef]

72. Ravet, K.; Pilon, M. Copper and iron homeostasis in plants: The challenges of oxidative stress. Antioxid. Redox Sign. 2013, 19, 919-932. [CrossRef] [PubMed]

73. Luo, Y.; Liu, Y.B.; Dong, Y.X.; Gao, X.Q.; Zhang, X.S. Expression of a putative alfalfa helicase increases tolerance to abiotic stress in Arabidopsis by enhancing the capacities for ROS scavenging and osmotic adjustment. J. Plant Physiol. 2009, 166, 385-394. [CrossRef] [PubMed]

74. Rakwal, R.; Yang, G.; Komatsu, S. Chitinase induced by jasmonic acid, methyl jasmonate, ethylene and protein phosphatase inhibitors in rice. Mol. Biol. Rep. 2004, 31, 113-119. [CrossRef]

75. Taddei, S.; Bernardi, R.; Salvini, M.; Pugliesi, C.; Durante, M. Effect of copper on callus growth and gene expression of in vitro-cultured pith explants of Nicotiana glauca. Plant Biosyst. 2007, 141, 194-203. [CrossRef]

76. Zhou, X.X.; Yang, L.T.; Qi, Y.P.; Guo, P.; Chen, L.S. Mechanisms on boron-induced alleviation of aluminum-toxicity in Citrus grandis seedlings at a transcriptional level revealed by cDNA-AFLP analysis. PLoS ONE 2015, 10, e0115485. [CrossRef]

77. Sudo, E.; Itouga, M.; Yoshida-Hatanaka, K.; Ono, Y.; Sakakibara, H. Gene expression and sensitivity in response to copper stress in rice leaves. J. Exp. Bot. 2008, 59, 3465-3474. [CrossRef] 
78. Huang, J.H.; Xu, J.; Ye, X.; Luo, T.Y.; Ren, L.H.; Fan, G.C.; Yi, P.Q.; Li, Q.; Ferarezi, R.H.; Chen, L.S. Magnesium deficiency affects secondary lignification of the vascular system in Citrus sinensis seedlings. Trees Struct. Funct. 2019, 33, 171-182. [CrossRef]

79. Ye, X.; Chen, X.F.; Deng, C.L.; Yang, L.T.; Lai, L.W.; Guo, J.X.; Chen, L.S. Magnesium-deficiency effects on pigments, photosynthesis and photosynthetic electron transport of leaves, and nutrients of leaf blades and veins in Citrus sinensis seedlings. Plants 2019, 8, 389. [CrossRef]

80. You, X.; Yang, L.T.; Lu, Y.B.; Li, H.; Zhang, S.Q.; Chen, L.S. Proteomic changes of Citrus roots in response to long-term manganese toxicity. Trees Struct. Funct. 2014, 28, 1383-1399. [CrossRef]

81. Bradford, M.M. A rapid and sensitive method for quantitation of microgramand quantities of protein utilizing the principle of protein-dye binding. Anal. Biochem. 1976, 72, 248-254. [CrossRef]

82. Sang, W.; Huang, Z.R.; Yang, L.T.; Guo, P.; Ye, X.; Chen, L.S. Effects of high toxic boron concentration on protein profiles in roots of two Citrus species differing in boron tolerance revealed by a 2-DE based MS approach. Front. Plant Sci. 2017, 8, 180. [CrossRef] [PubMed]

83. Peng, H.Y.; Qi, Y.P.; Lee, J.; Yang, L.T.; Guo, P.; Jiang, H.X.; Chen, L.S. Proteomic analysis of Citrus sinensis roots and leaves in response to long-term magnesium-deficiency. BMC Genom. 2015, 16, 253. [CrossRef] [PubMed]

84. Figueiredo, A.; Martins, J.; Sebastiana, M.; Guerreiro, A.; Silva, A.; Matos, A.R.; Monteiro, F.; Pais, M.S.; Roepstorff, P.; Coelho, A.V. Specific adjustments in grapevine leaf proteome discriminating resistant and susceptible grapevine genotypes to Plasmopara viticola. J. Proteome 2017, 152, 48-57. [CrossRef] [PubMed]

85. Zhang, J.; Li, Q.; Qi, Y.P.; Huang, W.L.; Yang, L.T.; Lai, N.W.; Ye, X.; Chen, L.S. Low pH-responsive proteins revealed by a 2-DE based MS approach and related physiological responses in Citrus leaves. BMC Plant Biol. 2019, 18, 188. [CrossRef]

86. Guo, P.; Qi, Y.P.; Huang, W.L.; Yang, L.T.; Huang, Z.R.; Lai, N.W.; Chen, L.S. Aluminum-responsive genes revealed by RNA-Seq and related physiological responses in leaves of two Citrus species with contrasting aluminum-tolerance. Ecotoxicol. Environ. Saf. 2018, 158, 213-222. [CrossRef]

87. Yang, L.T.; Qi, Y.P.; Lu, Y.B.; Guo, P.; Sang, W.; Feng, H.; Zhang, H.X.; Chen, L.S. iTRAQ protein profile analysis of Citrus sinensis roots in response to long-term boron-deficiency. J. Proteome 2013, 93, 179-206. [CrossRef]

88. Guo, P.; Qi, Y.P.; Cai, Y.T.; Yang, T.Y.; Yang, L.T.; Huang, Z.R.; Chen, L.S. Aluminum effects on photosynthesis, reactive oxygen species and methylglyoxal detoxification in two Citrus species differing in aluminum tolerance. Tree Physiol. 2018, 38, 1548-1565. [CrossRef] 